\title{
Synthesis, Reactivity and Properties of a Class of $\pi$-Extended BODIPY Derivatives
}

Wanwan Li, ${ }^{a}$ Qingbao Gong, ${ }^{a}$ Xing Guo,,${ }^{\mathrm{a}, *}$ Qinghua Wu, ${ }^{\mathrm{a}}$ Fei Chang, ${ }^{\mathrm{a}}$ Hua Wang, ${ }^{\mathrm{a}}$ Fan Zhang, ${ }^{\mathrm{a}}$ Erhong $\mathrm{Hao}^{\mathrm{a}, *}$ and Lijuan $\mathrm{Jiao}^{\mathrm{a}, *}$

${ }^{\text {a }}$ Laboratory of Functional Molecular Solids, Ministry of Education; School of Chemistry and Materials Science, Anhui Normal University, Wuhu, 241000, China.

E-mail: guoxing@ahnu.edu.cn, haoehong@ahnu.edu.cn, jiao421@ahnu.edu.cn

\section{Contents:}

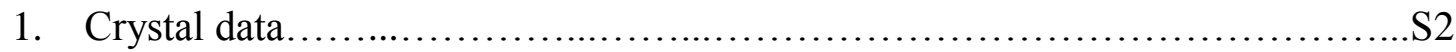

2. Table S5. Optimization for the yield of $2 \mathbf{a} \ldots \ldots \ldots \ldots \ldots \ldots \ldots \ldots \ldots \ldots \ldots$

3. Photophysical data................................................... 10

4. Electrochemical spectra........................................ 15

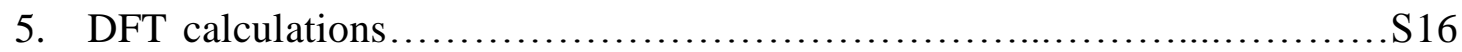

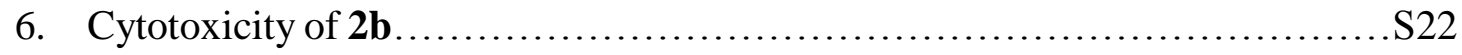

7. Copies of ${ }^{1} \mathrm{H}$ and ${ }^{13} \mathrm{C}$ NMR spectra for all new compounds...................S25

8. High resolution mass spectroscopes for all new compounds.................S34 


\section{Crystal data}

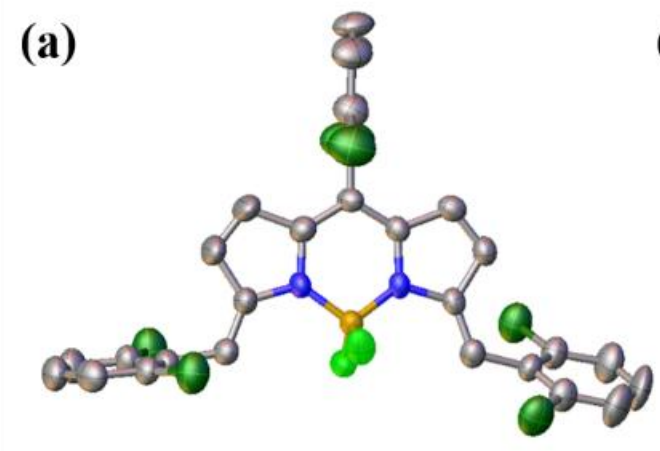

(b)

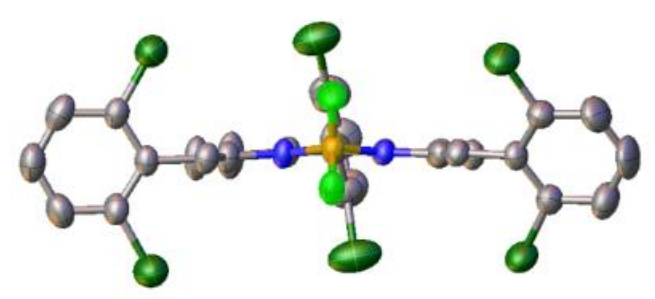

(c)
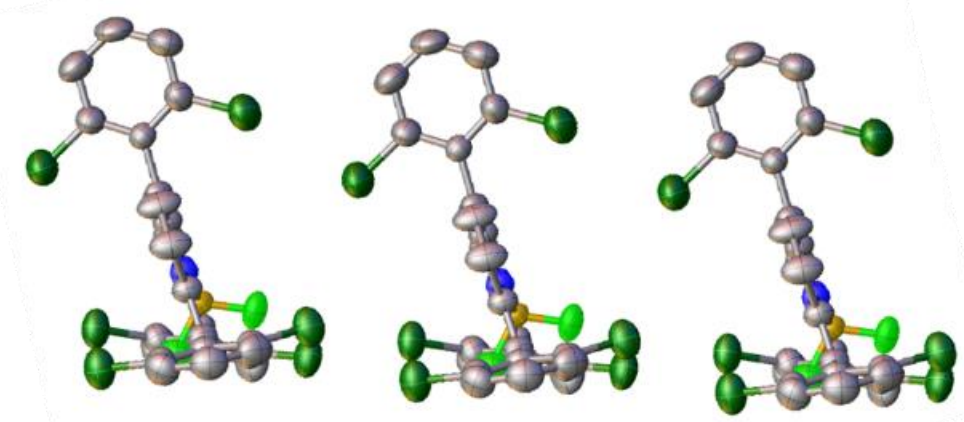

Figure S1. Single-crystal structure of $\mathbf{2 b}$, with thermal ellipsoids shown at $50 \%$ probability: (a) top view, (b) bottom view and (c) Crystal packing of X-ray structures of BODIPY 2b. C, gray; N, blue; B, dark yellow; F, light green; $\mathrm{Cl}$, dark green. Hydrogen atoms have removed for clarity. 
(a)

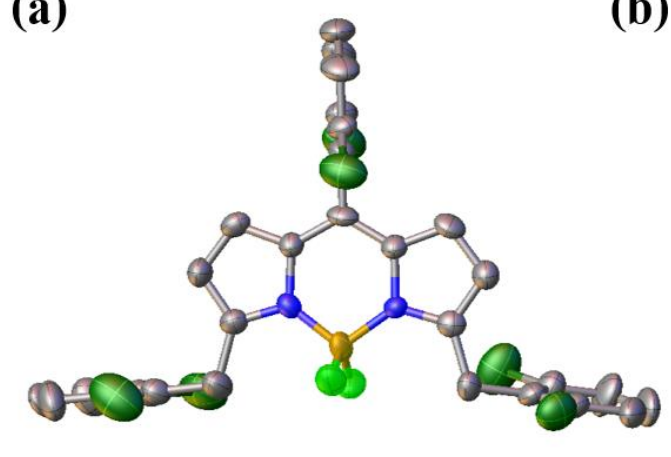

(c)

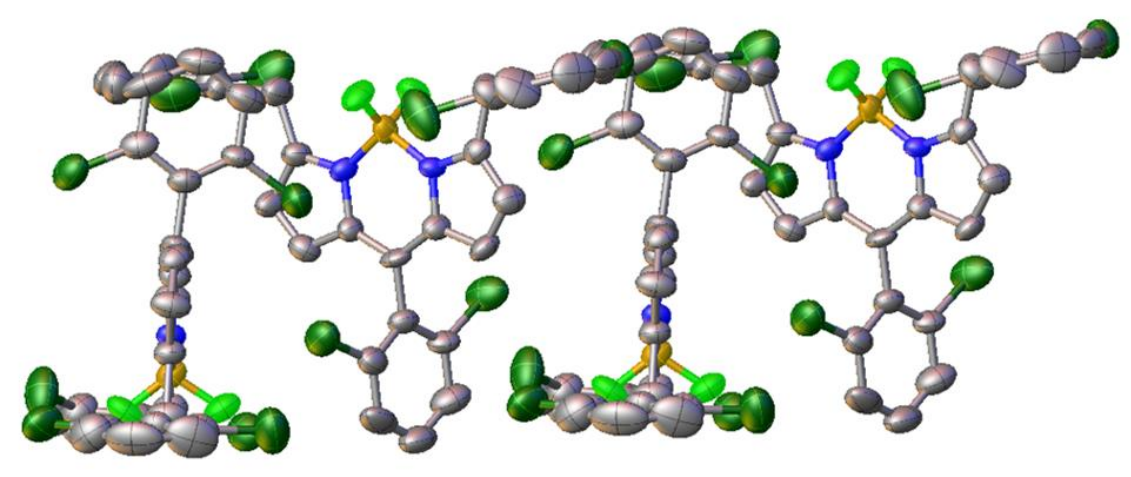

Figure S2. Single-crystal structure of 7, with thermal ellipsoids shown at 50\% probability: (a) top view, (b) bottom view and (c) Crystal packing of X-ray structures of BODIPY 7. C, gray; N, blue; B, dark yellow; F, light green; $\mathrm{Cl}$, dark green. Hydrogen atoms have removed for clarity. 
(a)

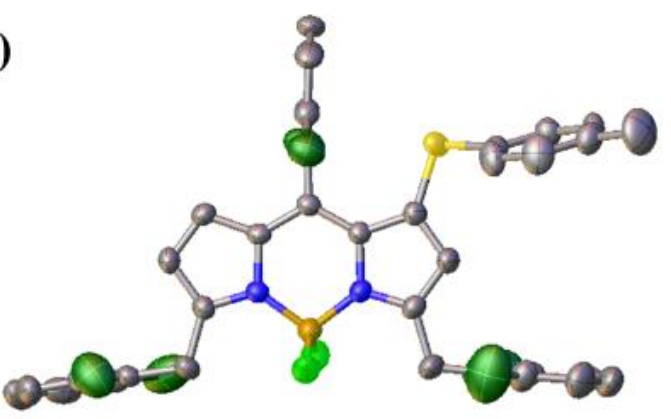

(b)

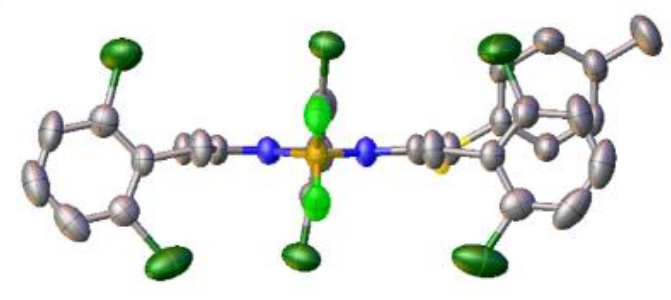

(c)

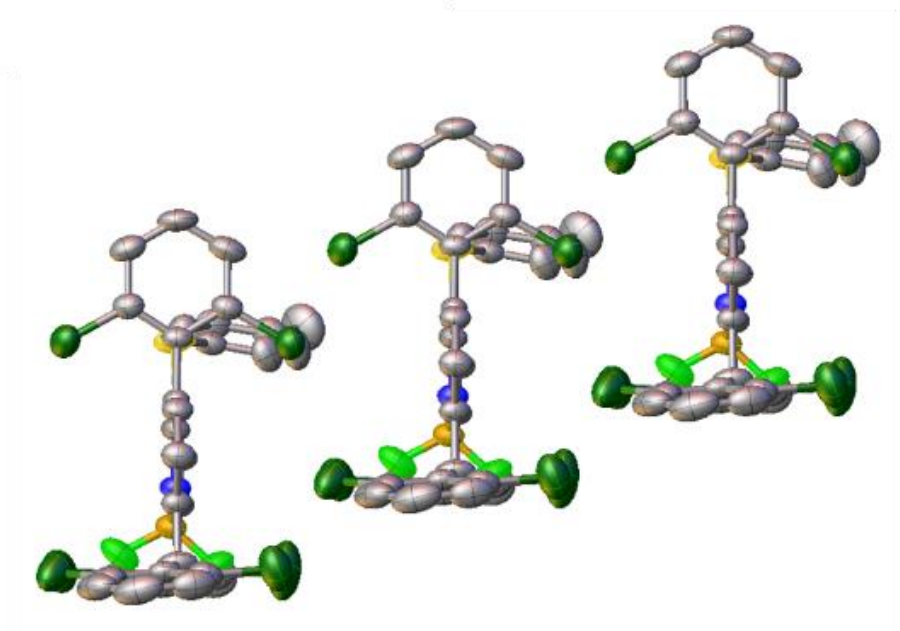

Figure S3. Single-crystal structure of $\mathbf{6 b}$, with thermal ellipsoids shown at 50\% probability: (a) top view, (b) side view and (c) Crystal packing of X-ray structures of BODIPY 6b. C, gray; N, blue; B, dark yellow; F, light green; $\mathrm{Cl}$, dark green; S, yellow. Hydrogen atoms have removed for clarity. 
Table S1. Selected Geometrical Parameters of 2b, 7 and $6 b$ obtained from crystallography
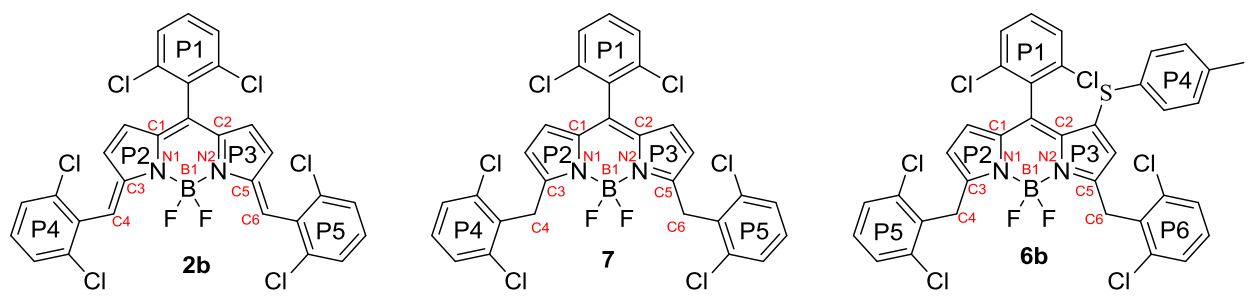

\begin{tabular}{|c|c|c|c|}
\hline & $2 \mathbf{b}$ & 7 & $6 b$ \\
\hline B1-N1 bond length $(\AA)$ & $1.5445(55)$ & $1.5484(113)$ & $1.5526(42)$ \\
\hline B1-N2 bond length $(\AA)$ & $1.5362(51)$ & $1.5277(87)$ & $1.5561(45)$ \\
\hline B-F bond length $(\AA)$ & $\begin{array}{l}1.3823(52) \\
1.3777(48)\end{array}$ & $\begin{array}{c}1.3715(88) \\
1.4013(100)\end{array}$ & $\begin{array}{l}1.3775(43) \\
1.3741(43)\end{array}$ \\
\hline C1-N1 bond length $(\AA)$ & $1.3633(52)$ & $1.3888(103)$ & $1.3891(39)$ \\
\hline C2-N2 bond length $(\AA)$ & $1.3657(52)$ & $1.3944(96)$ & $1.3941(35)$ \\
\hline C3-C4 bond length $(\AA)$ & $1.3278(59)$ & $1.4864(111)$ & $1.4937(45)$ \\
\hline C5-C6 bond length $(\AA)$ & $1.3432(61)$ & $1.4999(133)$ & $1.4958(45)$ \\
\hline $\mathrm{H} \cdots \mathrm{F}$ bond length $(\AA)$ & $\begin{array}{l}2.6401(23) \\
2.7559(24) \\
2.7923(25) \\
2.7016(22)\end{array}$ & $\begin{array}{l}2.7678(45) \\
2.4892(37) \\
2.4381(35)\end{array}$ & $\begin{array}{l}2.6181(23) \\
2.7802(20) \\
2.6643(22) \\
2.6697(20)\end{array}$ \\
\hline $\begin{array}{l}\text { dihedral angles between } \\
\text { P2 and P3 (deg) } \\
\text { dihedral angles between }\end{array}$ & $5.361(125)$ & $1.361(236)$ & 4.061(111) \\
\hline $\begin{array}{l}\text { P1 and BODIPY core } \\
\text { (deg) }\end{array}$ & $80.783(36)$ & 78.554(57) & $83.450(4)$ \\
\hline $\begin{array}{l}\text { dihedral angles between } \\
\text { P4 and BODIPY core } \\
\text { (deg) }\end{array}$ & $81.796(82)$ & $87.382(138)$ & $80.839(84)$ \\
\hline $\begin{array}{l}\text { dihedral angles between } \\
\text { P5 and BODIPY core } \\
\text { (deg) }\end{array}$ & $62.738(86)$ & $88.019(170)$ & $86.205(57)$ \\
\hline $\begin{array}{l}\text { dihedral angles between } \\
\text { P6 and BODIPY core } \\
\text { (deg) }\end{array}$ & l & l & $88.473(54)$ \\
\hline \multicolumn{4}{|c|}{$\begin{array}{l}\text { [a] BODIPY core: dipyyrin core }\left(\mathrm{C}_{9} \mathrm{~N}_{2} \mathrm{~B}\right) ;[\mathrm{b}] \mathbf{P 2} \text { and } \mathbf{P 3} \text { : pyrrole rings }\left(\mathrm{C}_{4} \mathrm{~N}\right) \text { in } \\
\text { dipyyrin core }\left(\mathrm{C}_{9} \mathrm{~N}_{2} \mathrm{~B}\right) \text {; the aryl groups } \mathbf{P 1}, \mathbf{P 4}, \mathbf{P 5} \text { in } \mathbf{2 b} \text { and } 7 \text { : } 2,6 \text {-dichlorophenyl } \\
\text { group }\left(\mathrm{C}_{6} \mathrm{Cl}_{2}\right) \text {; the aryl groups } \mathbf{P 1}, \mathbf{P 5}, \mathbf{P 6} \text { in } \mathbf{6 b}: 2,6 \text {-dichlorophenyl group }\left(\mathrm{C}_{6} \mathrm{Cl}_{2}\right) \text {; the } \\
\text { aryl group } \mathbf{P 4} \text { in } 6 \mathbf{6} \text { : } 4 \text {-methylphenyl }\left(\mathrm{C}_{7}\right) \text {. }\end{array}$} \\
\hline
\end{tabular}


Table S2. Crystal data and structure refinement for $\mathbf{2 b}$.

Empirical formula

Formula weight

Temperature/K

Crystal system

Space group

$\mathrm{a} / \AA$

$\mathrm{b} / \AA$

$\mathrm{c} / \AA$

$\alpha /{ }^{\circ}$

$\beta /{ }^{\circ}$

$\gamma /{ }^{\circ}$

Volume/ $\AA^{3}$

$\mathrm{Z}$

$\rho_{\text {calc }} / \mathrm{cm}^{3}$

$\mu / \mathrm{mm}^{-1}$

$\mathrm{F}(000)$

Crystal size $/ \mathrm{mm}^{3}$

Radiation

$2 \Theta$ range for data collection/ ${ }^{\circ}$

Index ranges

Reflections collected

Independent reflections

Data/restraints/parameters

Goodness-of-fit on $\mathrm{F}^{2}$

Final $\mathrm{R}$ indexes $[\mathrm{I}>=2 \sigma(\mathrm{I})]$

Final $\mathrm{R}$ indexes [all data]

Largest diff. peak/hole / e $\AA^{-3}$
$\mathrm{C}_{29} \mathrm{H}_{15} \mathrm{BCl}_{6} \mathrm{~F}_{2} \mathrm{~N}_{2}$

652.992

293.15

monoclinic

$\mathrm{P} 21 / \mathrm{c}$

14.1502(13)

31.189(3)

$14.8138(14)$

90

95.896(1)

90

6503.2(10)

8

1.334

0.561

2632.1

$0.23 \times 0.21 \times 0.2$

Mo $\mathrm{K} \alpha(\lambda=0.71073)$

2.62 to 50

$-18 \leq \mathrm{h} \leq 18,-40 \leq \mathrm{k} \leq 38,-19 \leq 1 \leq 18$

55633

$11446\left[\mathrm{R}_{\text {int }}=0.0806, \mathrm{R}_{\text {sigma }}=0.1164\right]$

$11446 / 0 / 721$

0.886

$\mathrm{R}_{1}=0.0583, \mathrm{wR}_{2}=0.1415$

$\mathrm{R}_{1}=0.1325, \mathrm{wR}_{2}=0.1691$

$0.56 /-0.56$ 
Table S3. Crystal data and structure refinement for 7.

Empirical formula

Formula weight

Temperature/K

Crystal system

Space group

$\mathrm{a} / \AA$

$\mathrm{b} / \AA$

$\mathrm{c} / \AA$

$\alpha /{ }^{\circ}$

$\beta /{ }^{\circ}$

$\gamma /{ }^{\circ}$

Volume $/ \AA^{3}$

$\mathrm{Z}$

$\rho_{\text {calc }} \mathrm{g} / \mathrm{cm}^{3}$

$\mu / \mathrm{mm}^{-1}$

$\mathrm{F}(000)$

Crystal size $/ \mathrm{mm}^{3}$

Radiation

$2 \Theta$ range for data collection/ ${ }^{\circ}$

Index ranges

Reflections collected

Independent reflections

Data/restraints/parameters

Goodness-of-fit on $\mathrm{F}^{2}$

Final R indexes $[\mathrm{I}>=2 \sigma(\mathrm{I})]$

Final R indexes [all data]

Largest diff. peak/hole / e $\AA^{-3}$

Flack parameter
$\mathrm{C}_{32} \mathrm{H}_{22} \mathrm{BCl}_{6} \mathrm{~F}_{2} \mathrm{~N}_{2}$

696.081

293.15

monoclinic

$\mathrm{P} 21$

$11.323(5)$

12.681(6)

$11.877(6)$

90

$110.731(7)$

90

1595.1(13)

2

1.449

0.577

708.0

$0.22 \times 0.21 \times 0.21$

$\operatorname{Mo} \mathrm{K} \alpha(\lambda=0.71073)$

3.84 to 50

$-13 \leq \mathrm{h} \leq 13,-15 \leq \mathrm{k} \leq 15,-13 \leq 1 \leq 14$

11403

$5435\left[\mathrm{R}_{\text {int }}=0.1200, \mathrm{R}_{\text {sigma }}=0.1714\right]$

$5435 / 1 / 361$

0.917

$\mathrm{R}_{1}=0.0672, \mathrm{wR}_{2}=0.1695$

$\mathrm{R}_{1}=0.1439, \mathrm{wR}_{2}=0.2014$

$0.50 /-0.47$

$0.1(3)$ 
Table S4. Crystal data and structure refinement for $6 \mathbf{b}$.

Empirical formula

Formula weight

Temperature/K

Crystal system

Space group

$\mathrm{a} / \AA ̊$

$\mathrm{b} / \AA ̊ \AA$

c/Å

$\alpha /{ }^{\circ}$

$\beta /{ }^{\circ}$

$\gamma /{ }^{\circ}$

Volume/ $\AA^{3}$

Z

$\rho_{\text {calcg }} / \mathrm{cm}^{3}$

$\mu / \mathrm{mm}^{-1}$

$\mathrm{F}(000)$

Crystal size $/ \mathrm{mm}^{3}$

Radiation

$2 \Theta$ range for data collection/ ${ }^{\circ}$

Index ranges

Reflections collected

Independent reflections

Data/restraints/parameters

Goodness-of-fit on $\mathrm{F}^{2}$

Final $\mathrm{R}$ indexes $[\mathrm{I}>=2 \sigma(\mathrm{I})]$

Final $\mathrm{R}$ indexes [all data]

Largest diff. peak/hole / e $\AA^{-3}$

Flack parameter
$\mathrm{C}_{36} \mathrm{H}_{23} \mathrm{BN}_{2} \mathrm{~F}_{2} \mathrm{SCl}_{6}$

777.13

293(2)

monoclinic

$\mathrm{P} 21$

$7.6749(5)$

$12.2773(8)$

$18.7150(13)$

90

$94.7790(10)$

90

1757.3(2)

2

1.469

0.589

788.0

$0.230 \times 0.210 \times 0.200$

$\operatorname{MoK} \alpha(\lambda=0.71073)$

2.184 to 50.702

$-9 \leq \mathrm{h} \leq 9,-14 \leq \mathrm{k} \leq 14,-22 \leq 1 \leq 22$

17871

$6421\left[R_{\text {int }}=0.0290, R_{\text {sigma }}=0.0359\right]$

$6421 / 1 / 434$

1.028

$\mathrm{R}_{1}=0.0419, \mathrm{wR}_{2}=0.0956$

$\mathrm{R}_{1}=0.0549, \mathrm{wR}_{2}=0.1045$

$0.45 /-0.32$

$0.01(2)$ 
2. Table S5. Optimization for the yield of $2 a .^{\text {a }}$

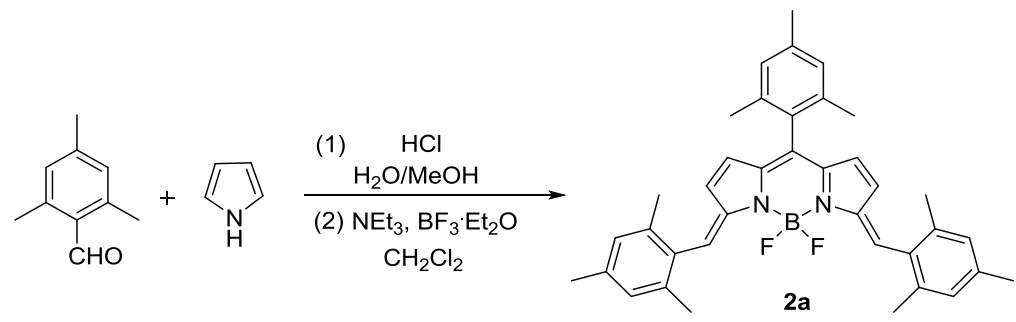

\begin{tabular}{cccccc}
\hline entries & $\begin{array}{c}\text { mesitaldehyde } \\
(\mathrm{mM})\end{array}$ & $\begin{array}{c}\text { pyrrole } \\
(\text { equiv })\end{array}$ & $\begin{array}{c}\mathrm{HCl} \\
(\mathrm{mM})\end{array}$ & $\begin{array}{c}\mathrm{H}_{2} \mathrm{O} / \mathrm{MeOH} \\
(\mathrm{v} / \mathrm{v})\end{array}$ & $\begin{array}{c}\text { yield }^{\mathrm{b}} \\
(\%)\end{array}$ \\
\hline 1 & 230 & 0.65 & 0.08 & $4 / 1$ & 5 \\
2 & 230 & 0.7 & 0.08 & $4 / 1$ & 7 \\
3 & 230 & 1 & 0.08 & $4 / 1$ & 3 \\
4 & 230 & 2 & 0.08 & $4 / 1$ & 2 \\
5 & 230 & 0.7 & 0.08 & $3 / 1$ & 10 \\
6 & 230 & 0.7 & 0.08 & $2 / 1$ & 5 \\
7 & 230 & 0.7 & 0.08 & $3 / 2$ & $\mathrm{NR}$ \\
8 & 25 & 0.7 & 0.08 & $3 / 1$ & 4 \\
9 & 25 & 0.7 & 0.25 & $3 / 1$ & 3 \\
\hline
\end{tabular}

${ }^{a}$ All reactions were performed under the following constant conditions: first step, $\mathrm{MeOH} / \mathrm{H}_{2} \mathrm{O}=\mathrm{v} / \mathrm{v}, 20 \mathrm{~h}$, room temperature; second step, $\mathrm{CH}_{2} \mathrm{Cl}_{2}, \mathrm{NEt}_{3}$ (7.9 equiv), $\mathrm{BF}_{3} \cdot \mathrm{OEt}_{2}$ (9.3 equiv), $6 \mathrm{~h}$, room temperature. ${ }^{\mathrm{b}}$ Isolated yields based on mesitaldehyde. $\mathrm{NR}=$ no reaction. 


\section{Photophysical data}

Table S6 Photophysical properties of $2 \mathrm{a}, 2 \mathrm{~b}$, and 7 in different solvents at room temperature.

\begin{tabular}{|c|c|c|c|c|c|c|}
\hline entry & solvent & $\lambda_{\mathrm{abs}}{ }^{\max }(\mathrm{nm})$ & $\varepsilon^{[\mathrm{a}]}$ & $\lambda_{\mathrm{em}}^{\max }(\mathrm{nm})$ & $\Phi^{[\mathrm{b}]}$ & $\begin{array}{c}\text { Stokes-shift } \\
\quad\left(\mathrm{cm}^{-1}\right)\end{array}$ \\
\hline \multirow{5}{*}{$2 \mathbf{a}$} & Hexane & 570 & 41 & 635 & 0.02 & 1800 \\
\hline & Toluene & 571 & 39 & 642 & 0.01 & 1940 \\
\hline & Dichloromethane & 568 & 35 & 638 & 0.01 & 1930 \\
\hline & $\mathrm{N}, \mathrm{N}$-Dimethylformamide & 565 & 31 & 643 & 0.004 & 2150 \\
\hline & Acetonitrile & 563 & 39 & 637 & 0.003 & 2060 \\
\hline \multirow{5}{*}{$2 \mathbf{b}$} & Hexane & 563 & 42 & 612 & 0.17 & 1420 \\
\hline & Toluene & 568 & 38 & 617 & 0.18 & 1400 \\
\hline & Dichloromethane & 565 & 45 & 615 & 0.17 & 1440 \\
\hline & $\mathrm{N}, \mathrm{N}$-Dimethylformamide & 562 & 41 & 616 & 0.14 & 1560 \\
\hline & Acetonitrile & 555 & 39 & 608 & 0.16 & 1570 \\
\hline \multirow{5}{*}{7} & Hexane & 528 & 116 & 543 & 0.92 & 520 \\
\hline & Toluene & 530 & 109 & 544 & 0.91 & 490 \\
\hline & Dichloromethane & 529 & 94 & 543 & 0.95 & 490 \\
\hline & $\mathrm{N}, \mathrm{N}$-Dimethylformamide & 526 & 86 & 540 & 0.50 & 490 \\
\hline & Acetonitrile & 524 & 98 & 538 & 0.96 & 500 \\
\hline
\end{tabular}

[a] Molar extinction coefficient $\left[10^{3} \mathrm{M}^{-1} \mathrm{~cm}^{-1}\right]$. [b] Fluorescence quantum yields of compound 7 were calculated using Rhodamine B $(\Phi=0.49$ in ethanol) as the reference. The fluorescence quantum yields of $\mathbf{2 a}$ and $\mathbf{2 b}$ were calculated using Cresyl Violet perchlorate $(\Phi=0.54$ in methanol) as the reference. 

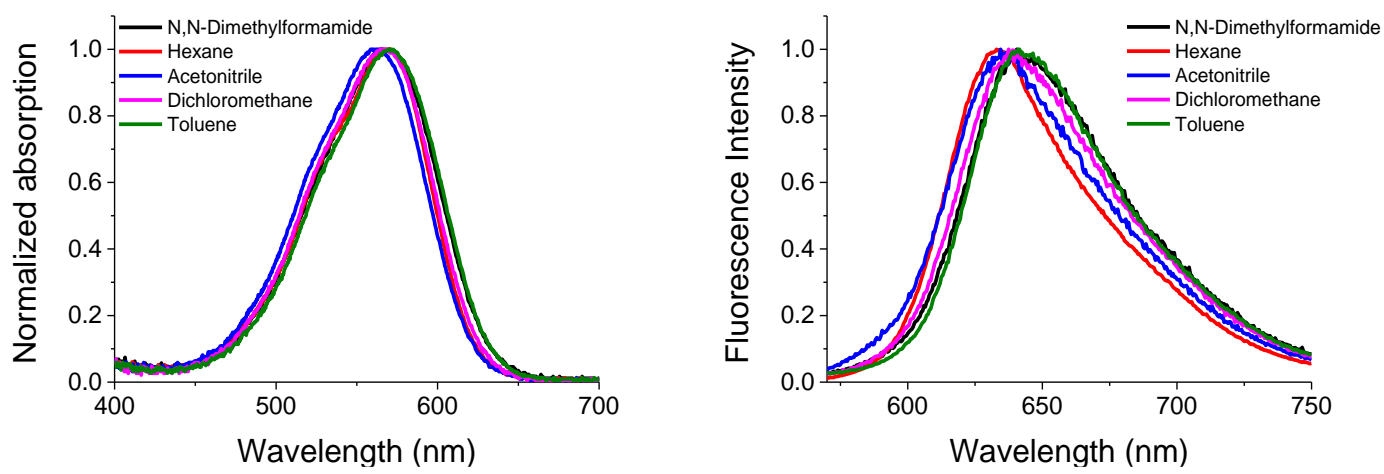

Figure S4. Normalized absorption (left) and emission (right) spectra of Ex-BODIPY $2 \mathbf{a}$ recorded in different solvents, excited at $520 \mathrm{~nm}$.
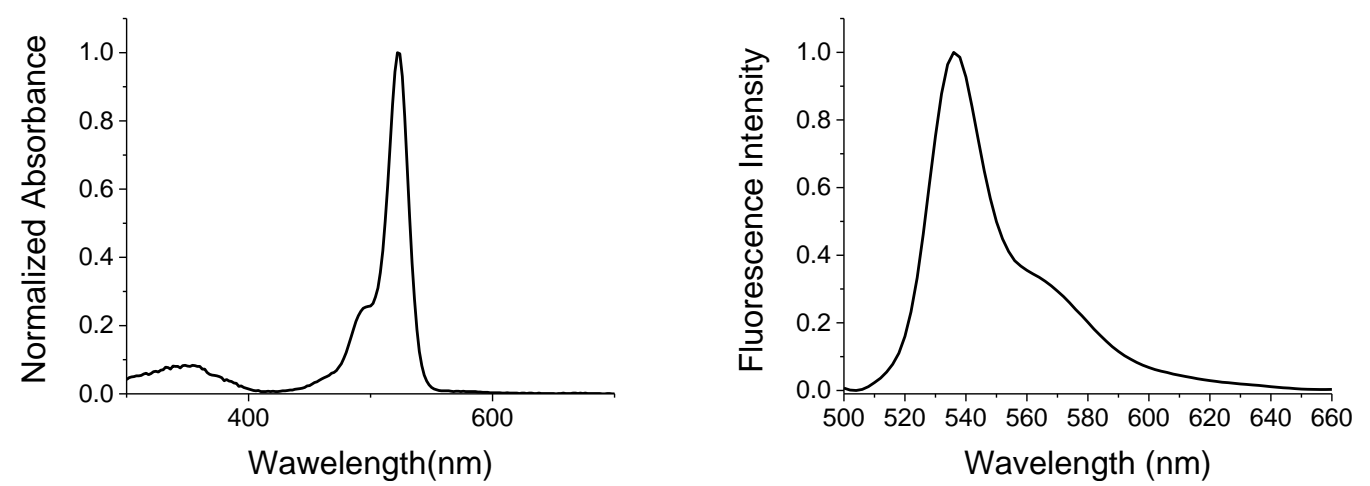

Figure S5. Normalized absorption (left) and emission (right) spectra of BODIPY 3a recorded in dichloromethane, excited at $490 \mathrm{~nm}$.
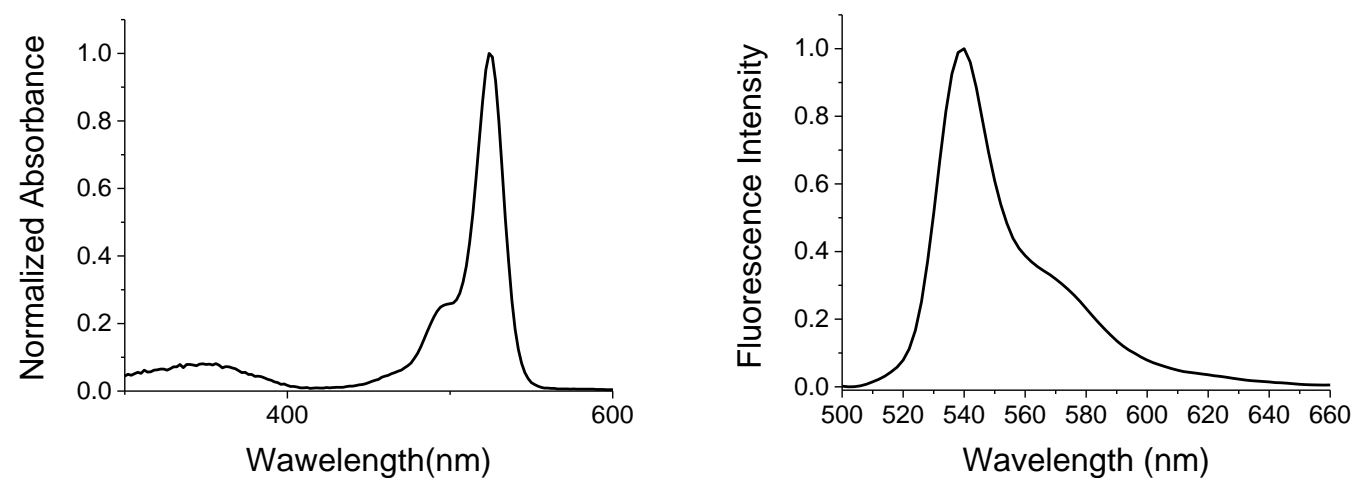

Figure S6. Normalized absorption (left) and emission (right) spectra of BODIPY 4a recorded in dichloromethane, excited at $490 \mathrm{~nm}$. 

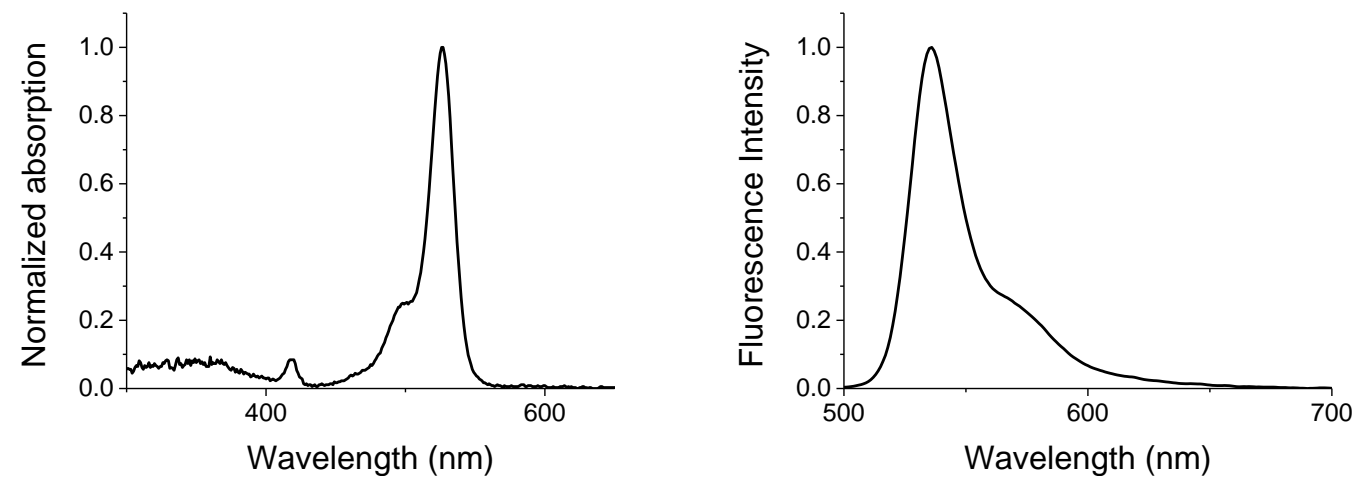

Figure S7. Normalized absorption (left) and emission (right) spectra of BODIPY 5a recorded in dichloromethane, excited at $490 \mathrm{~nm}$.
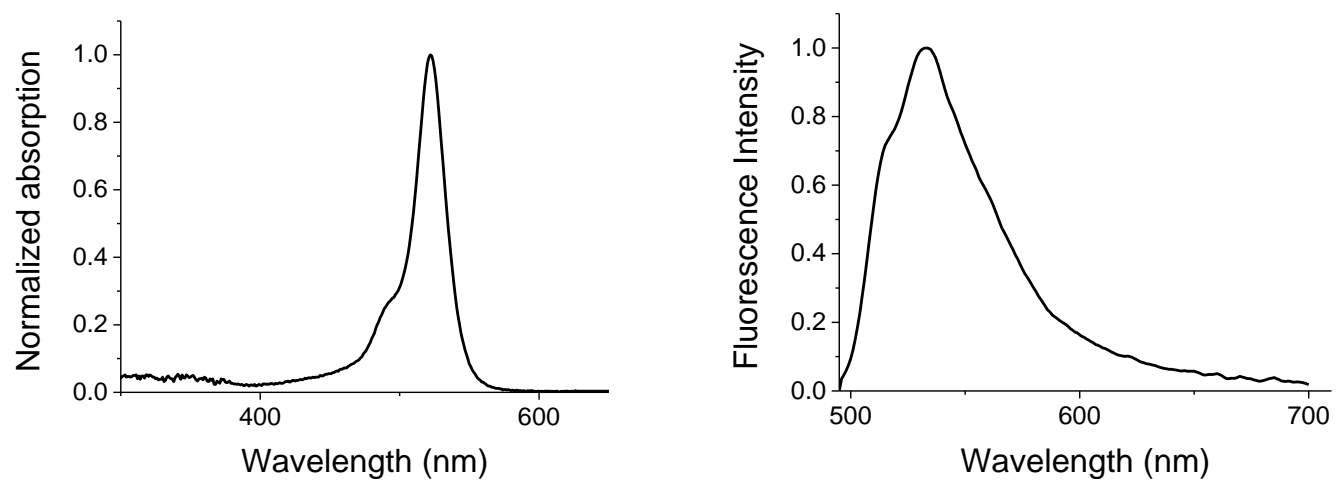

Figure S8. Normalized absorption (left) and emission (right) spectra of BODIPY 6a recorded in dichloromethane, excited at $480 \mathrm{~nm}$.
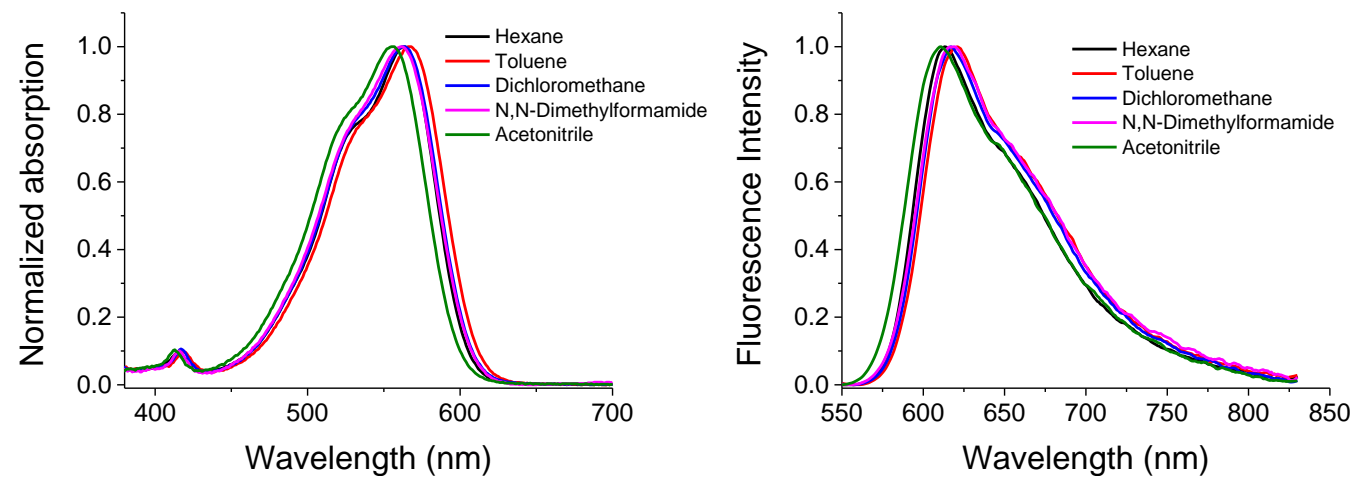

Figure S9. Normalized absorption (left) and emission (right) spectra of Ex-BODIPY $\mathbf{2 b}$ recorded in different solvents, excited at $530 \mathrm{~nm}$. 

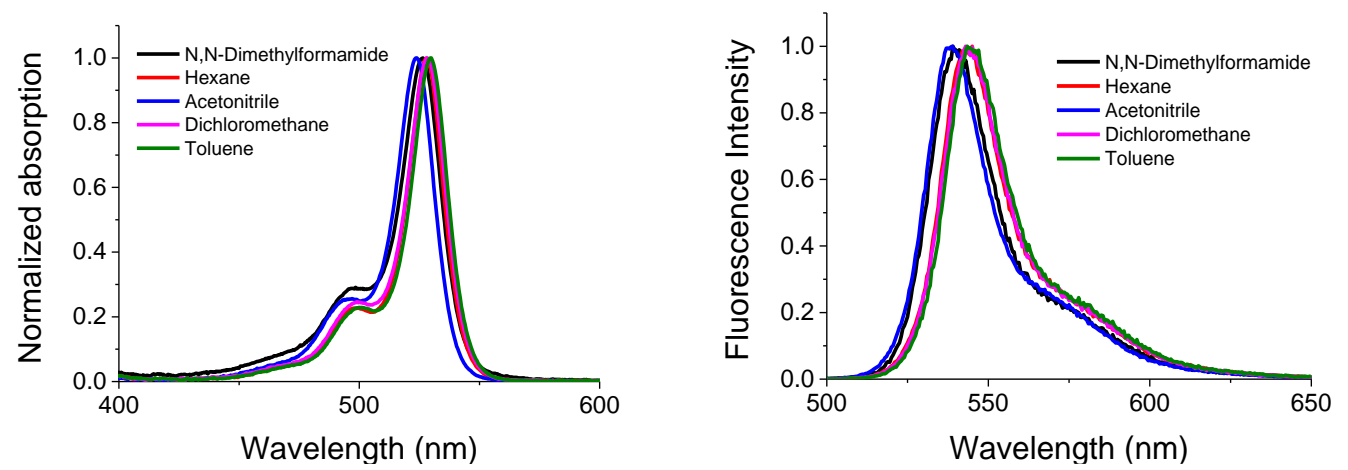

Figure S10. Normalized absorption (left) and emission (right) spectra of BODIPY 7 recorded in dichloromethane, excited at $490 \mathrm{~nm}$.
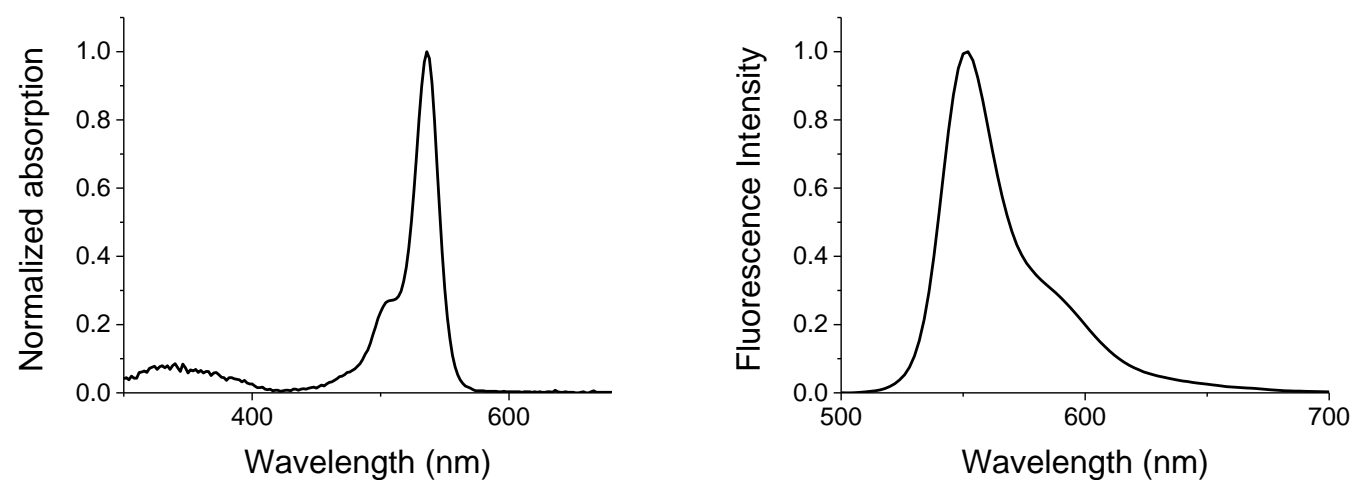

Figure S11. Normalized absorption (left) and emission (right) spectra of BODIPY $\mathbf{4 b}$ recorded in dichloromethane, excited at $490 \mathrm{~nm}$.
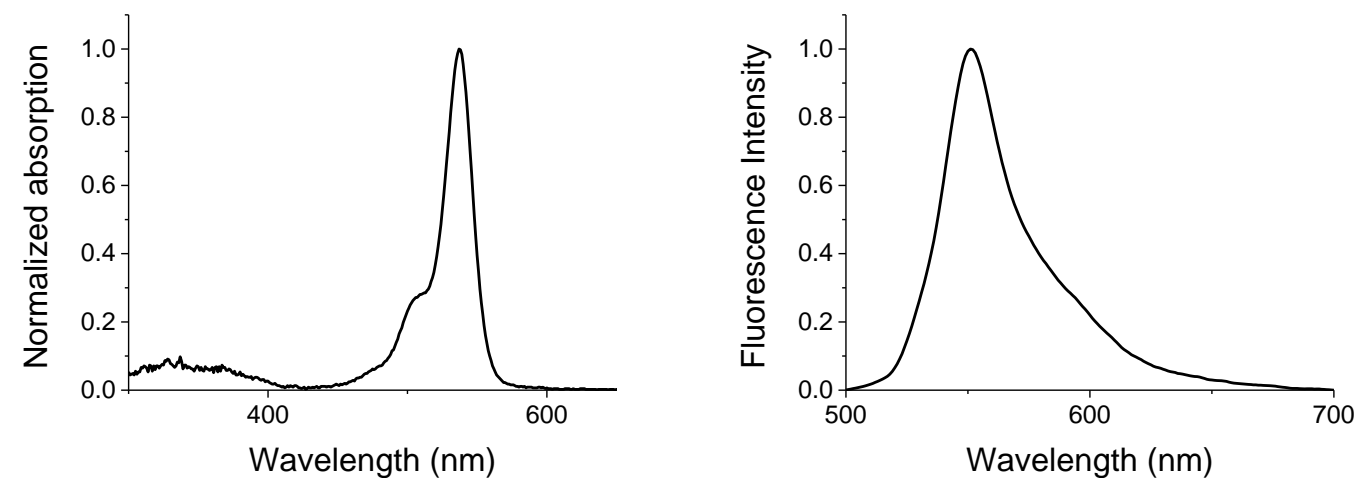

Figure S12. Normalized absorption (left) and emission (right) spectra of BODIPY $\mathbf{5 b}$ recorded in dichloromethane, excited at $490 \mathrm{~nm}$. 

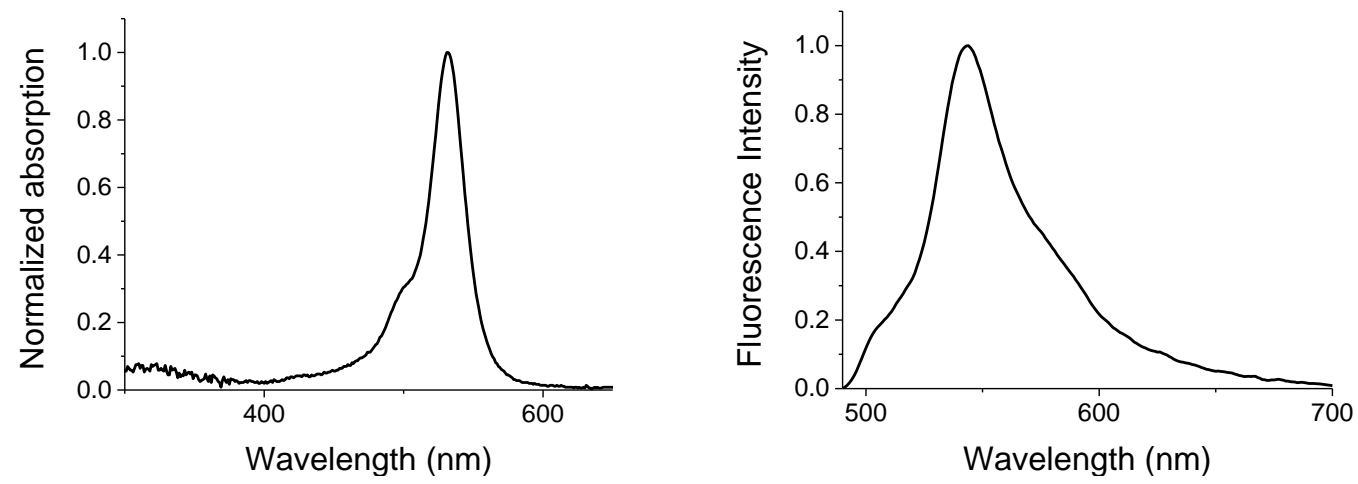

Figure S13. Normalized absorption (left) and emission (right) spectra of BODIPY $6 \mathbf{b}$ recorded in dichloromethane, excited at $480 \mathrm{~nm}$.
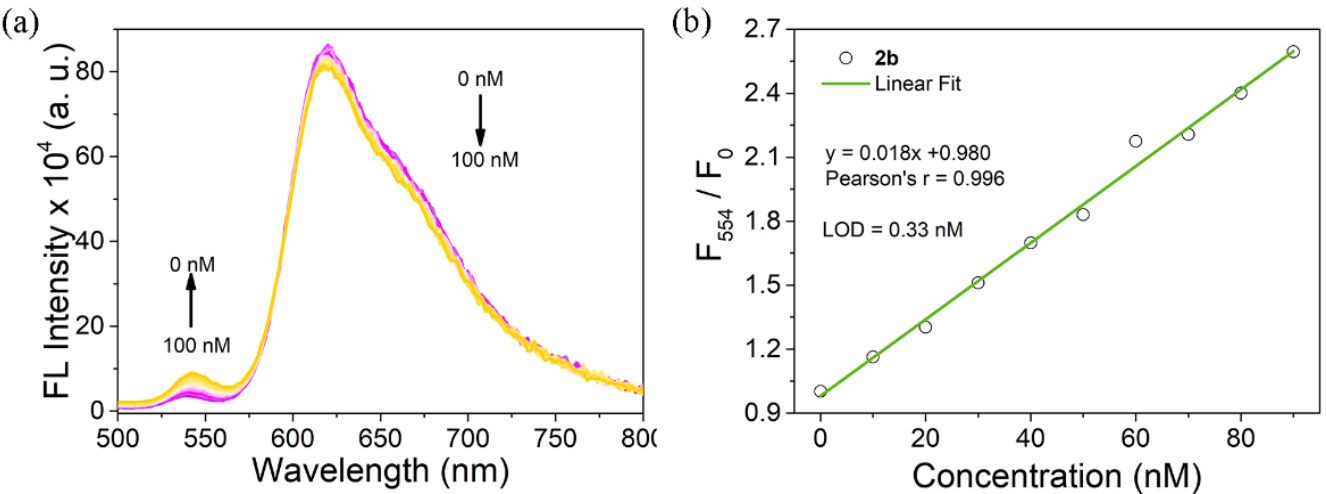

Figure S14. (a) Fluorescence spectra of $\mathbf{2 b}(20 \mu \mathrm{M})$ in DMSO/PBS buffer mixture upon additions of various concentrations of GSH $(0-100 \mathrm{nM})$, excitation at $480 \mathrm{~nm}$. (b) Plot of the fluorescence intensity ratio $\left(\mathrm{F} / \mathrm{F}_{0}\right.$ at $\left.554 \mathrm{~nm}\right)$ vs the concentration of GSH. The limit of detection (LOD) for GSH was calculated to be $0.33 \mathrm{nM}$ in the term of LOD = $3 \delta / \mathrm{k}$, where $\delta$ is the standard deviation of blank measurements, $\mathrm{k}$ is the slop of the line.

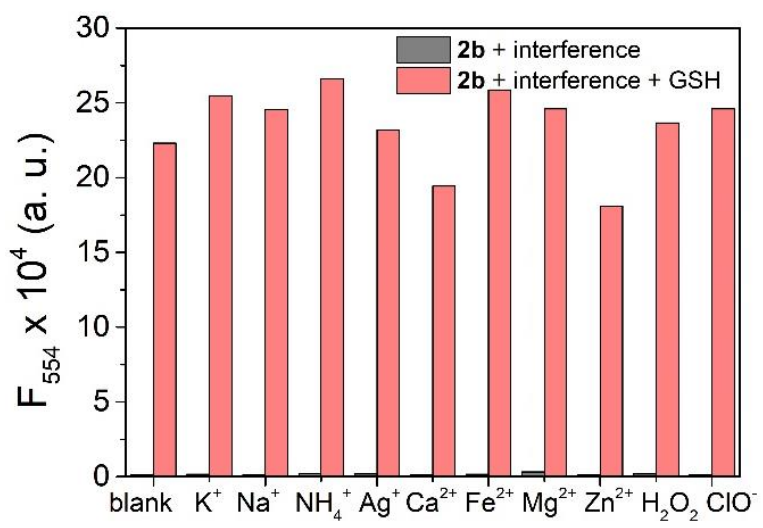

Figure S15. Emission spectral response of $\mathbf{2 b}(20 \mu \mathrm{M})$ in DMSO-PBS buffer solution $(60: 40, \mathrm{v} / \mathrm{v})$ upon addition of different species. $\mathrm{F}_{554}$ represent the emission intensity at $554 \mathrm{~nm}$. The species $(1 \mathrm{mM})$ used were blank, $\mathrm{KCl}, \mathrm{Na}_{2} \mathrm{CO}_{3}, \mathrm{CH}_{3} \mathrm{COONH}_{4}$, $\mathrm{CH}_{3} \mathrm{COOAg}, \mathrm{CaCl}_{2}, \mathrm{FeSO}_{4}, \mathrm{MgSO}_{4}, \mathrm{ZnSO}_{4}, \mathrm{H}_{2} \mathrm{O}_{2}$ and $\mathrm{NaClO}$. The excitation wavelength was $480 \mathrm{~nm}$. 


\section{Electrochemical spectra}

Table S7. Electrochemical data acquired at $100 \mathrm{mV} \mathrm{s}^{-1}$, and HOMO-LUMO Gaps determined from spectroscopy of dyes $2 a, 2 b, 7$ and 8 .
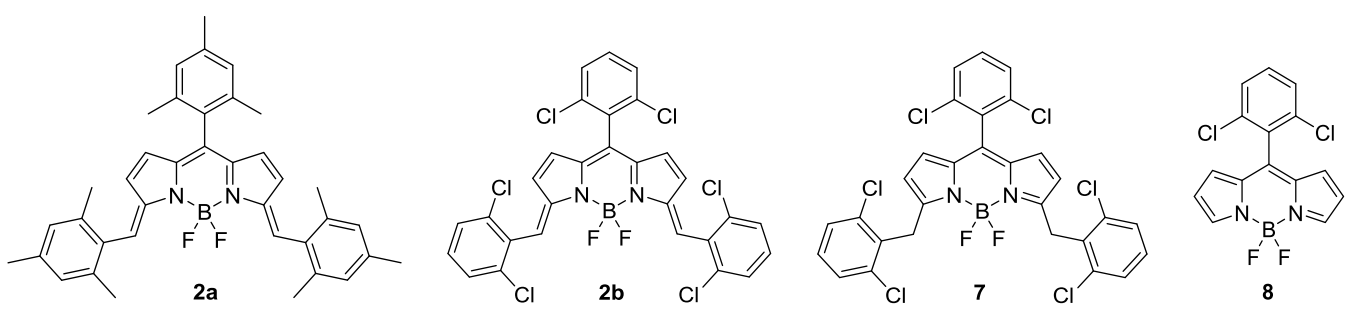

\begin{tabular}{|c|c|c|c|c|c|c|c|c|c|c|}
\hline dyes & $\begin{array}{c}E_{\mathrm{B} / \mathrm{B}^{-}} \\
(\mathrm{V})\end{array}$ & $\begin{array}{c}E_{\mathrm{p} 2}{ }^{\mathrm{red}} \\
(\mathrm{V})\end{array}$ & $\begin{array}{c}E_{\mathrm{p} 2}{ }^{\mathrm{OX}} \\
(\mathrm{V})\end{array}$ & $\begin{array}{c}E_{\text {red }}{ }^{\text {onset }} \\
(\mathrm{V})\end{array}$ & $\begin{array}{c}E^{o} \mathrm{~B}^{+} \cdot \mathrm{B} \\
(\mathrm{V})\end{array}$ & $\begin{array}{c}E_{\text {ox }}{ }^{\text {onset }} \\
(\mathrm{V})\end{array}$ & $\begin{array}{c}\text { LUMO } \\
(\mathrm{eV})\end{array}$ & $\begin{array}{c}\text { HOMO } \\
(\mathrm{eV})\end{array}$ & $\begin{array}{l}E_{\mathrm{g}}^{\mathrm{e}} \\
(\mathrm{eV})\end{array}$ & $\begin{array}{l}E_{\mathrm{g}}{ }^{\mathrm{O}} \\
(\mathrm{eV})\end{array}$ \\
\hline $2 a$ & -1.23 & -1.88 & 1.39 & -1.13 & 1.02 & 0.87 & -3.27 & -5.27 & 2.00 & 1.98 \\
\hline $2 b$ & -0.91 & -1.47 & 1.53 & -0.78 & 1 & 1.25 & -3.62 & -5.65 & 2.03 & 2.04 \\
\hline 7 & -1.01 & 1 & 1.59 & -0.91 & 1 & 1.35 & -3.49 & -5.75 & 2.26 & 2.28 \\
\hline 8 & -0.85 & 1 & 1 & -0.51 & 1 & 1 & -3.89 & 1 & 1 & 2.28 \\
\hline \multicolumn{11}{|c|}{$\begin{array}{l}E_{\mathrm{p} 2}{ }^{\text {red }}=\text { irreversible reduction peak potentials; } E_{\mathrm{p} 2}{ }^{\mathrm{OX}}=\text { irreversible oxidation peak } \\
\text { potentials; } E_{\mathrm{B} / \mathrm{B}}{ }^{-}=\text {reversible reduction potential; } E_{\mathrm{B}}^{o}{ }^{+} \cdot{ }_{\mathrm{B}}=\text { reversible oxidation potential } \\
E_{\mathrm{red}}{ }^{\text {onset }}=\text { the onset reduction potentials; } E_{\mathrm{ox}}{ }^{\text {onset }}=\text { the onset oxidation potentials; } E_{\mathrm{LUMO}} \\
=-\mathrm{e}\left(E_{\mathrm{red}}{ }^{\text {onset }}+4.4\right) ; E_{\mathrm{HOMO}}=-\mathrm{e}\left(E_{\mathrm{ox}}{ }^{\text {onset }}+4.4\right) ; E_{\mathrm{g}}{ }^{\mathrm{e}}=\text { bandgap, obtained from the } \\
\text { intercept of the electrochemical data; } E_{\mathrm{g}}{ }^{\mathrm{e}}=E_{\mathrm{LUMO}}-E_{\mathrm{HOMO}} ; E_{\mathrm{g}}{ }^{\mathrm{o}}=\text { bandgap, obtained } \\
\text { from the intercept of the absorption spectra. }\end{array}$} \\
\hline
\end{tabular}




\section{DFT calculations}

PCM-DFT optimized coordinates for $2 a, 2 b, 7$ and 8 .

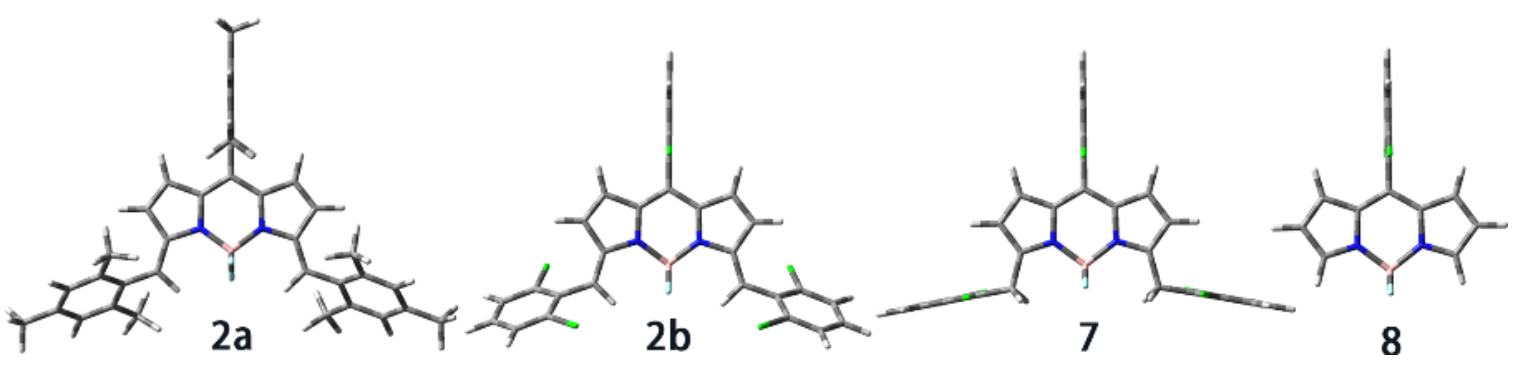

Figure S16. Optimized structures for $\mathbf{2 a}, \mathbf{2 b}, 7$ and $\mathbf{8 .}$

Compound 2a, optimized $\mathrm{S}_{0}$ state Geometry.

$\begin{array}{lrrr}\text { B } & 10.67910000 & 11.55710000 & 0.67190000 \\ \mathrm{C} & 11.54620000 & 9.83010000 & -1.11990000 \\ \mathrm{C} & 12.74680000 & 9.00550000 & -1.24960000 \\ \mathrm{H} & 12.94380000 & 8.44600000 & -1.96570000 \\ \mathrm{C} & 13.50890000 & 9.18770000 & -0.17540000 \\ \mathrm{H} & 14.33580000 & 8.79220000 & -0.01620000 \\ \mathrm{C} & 12.82450000 & 10.11120000 & 0.69700000 \\ \mathrm{C} & 13.22750000 & 10.55590000 & 1.94660000 \\ \mathrm{C} & 12.42970000 & 11.46230000 & 2.60230000 \\ \mathrm{C} & 12.57730000 & 12.01770000 & 3.92700000 \\ \mathrm{H} & 13.28240000 & 11.85810000 & 4.51200000 \\ \mathrm{C} & 11.52120000 & 12.80060000 & 4.16420000 \\ \mathrm{H} & 11.37520000 & 13.27720000 & 4.94820000 \\ \mathrm{C} & 10.64200000 & 12.79530000 & 3.01780000 \\ \mathrm{C} & 10.50800000 & 9.93430000 & -1.95980000 \\ \mathrm{C} & 10.37070000 & 9.14210000 & -3.19320000 \\ \mathrm{C} & 10.18570000 & 9.74750000 & -4.41620000 \\ \mathrm{C} & 10.08640000 & 9.06790000 & -5.59210000 \\ \mathrm{H} & 9.96950000 & 9.52510000 & -6.39220000 \\ \mathrm{C} & 10.16160000 & 7.69840000 & -5.58030000 \\ \mathrm{H} & 10.10940000 & 7.22030000 & -6.37600000 \\ \mathrm{C} & 10.31370000 & 7.03120000 & -4.38080000 \\ \mathrm{H} & 10.34270000 & 6.10060000 & -4.36020000 \\ \mathrm{C} & 10.42370000 & 7.75980000 & -3.21230000 \\ \mathrm{C} & 14.42620000 & 9.99950000 & 2.64350000 \\ \mathrm{C} & 14.43570000 & 8.73600000 & 3.16810000 \\ \mathrm{C} & 15.45440000 & 8.24010000 & 3.96380000 \\ \mathrm{H} & 15.40980000 & 7.38560000 & 4.32780000 \\ \mathrm{C} & 16.53160000 & 9.05420000 & 4.19370000 \\ \mathrm{C} & 16.66300000 & 10.27990000 & 3.62050000 \\ \mathrm{H} & 17.43440000 & 10.78520000 & 3.74130000 \\ & & 516 & \\ & & & \\ & & \end{array}$




\begin{tabular}{|c|c|c|c|}
\hline $\mathrm{C}$ & 15.59480000 & 10.75860000 & 2.83800000 \\
\hline $\mathrm{C}$ & 9.47690000 & 13.40690000 & 2.88670000 \\
\hline $\mathrm{C}$ & 8.88540000 & 14.20220000 & 3.98300000 \\
\hline $\mathrm{C}$ & 8.41570000 & 13.62870000 & 5.16030000 \\
\hline $\mathrm{C}$ & 7.90450000 & 14.38060000 & 6.20510000 \\
\hline $\mathrm{H}$ & 7.59630000 & 13.97580000 & 6.98310000 \\
\hline $\mathrm{C}$ & 7.86500000 & 15.75540000 & 6.05330000 \\
\hline $\mathrm{H}$ & 7.54380000 & 16.27750000 & 6.75180000 \\
\hline $\mathrm{C}$ & 8.28090000 & 16.36020000 & 4.91720000 \\
\hline $\mathrm{H}$ & 8.22400000 & 17.28490000 & 4.82440000 \\
\hline $\mathrm{C}$ & 8.79070000 & 15.58110000 & 3.89900000 \\
\hline $\mathrm{F}$ & 9.44390000 & 10.98380000 & 0.82270000 \\
\hline $\mathrm{F}$ & 10.57150000 & 12.65060000 & -0.13600000 \\
\hline $\mathrm{N}$ & 11.67900000 & 10.52500000 & 0.10170000 \\
\hline $\mathrm{N}$ & 11.25810000 & 11.93790000 & 2.06740000 \\
\hline $\mathrm{C}$ & 13.23942391 & 7.79908372 & 2.91767753 \\
\hline $\mathrm{H}$ & 12.32747494 & 8.34792394 & 3.02732919 \\
\hline $\mathrm{H}$ & 13.29932740 & 7.40136884 & 1.92614617 \\
\hline $\mathrm{H}$ & 13.26029093 & 6.99698434 & 3.62556251 \\
\hline $\mathrm{C}$ & 15.69531008 & 12.12902992 & 2.14271621 \\
\hline $\mathrm{H}$ & 15.31982028 & 12.05059024 & 1.14383953 \\
\hline $\mathrm{H}$ & 15.11750177 & 12.84761398 & 2.68555551 \\
\hline $\mathrm{H}$ & 16.71844312 & 12.44106697 & 2.11566701 \\
\hline $\mathrm{C}$ & 17.64043816 & 8.56346252 & 5.14304433 \\
\hline $\mathrm{H}$ & 17.21310695 & 7.93382064 & 5.89526714 \\
\hline $\mathrm{H}$ & 18.36787267 & 8.01060614 & 4.58618810 \\
\hline $\mathrm{H}$ & 18.11076105 & 9.40499400 & 5.60728687 \\
\hline $\mathrm{C}$ & 8.41810773 & 12.09756388 & 5.32527390 \\
\hline $\mathrm{H}$ & 8.14305733 & 11.63856397 & 4.39868534 \\
\hline $\mathrm{H}$ & 9.39688298 & 11.77088081 & 5.60842965 \\
\hline $\mathrm{H}$ & 7.71605574 & 11.81940598 & 6.08333141 \\
\hline $\mathrm{C}$ & 9.29170815 & 16.27614941 & 2.61935272 \\
\hline $\mathrm{H}$ & 10.35080480 & 16.14884868 & 2.53563401 \\
\hline $\mathrm{H}$ & 8.81188088 & 15.84270715 & 1.76683082 \\
\hline $\mathrm{H}$ & 9.06054168 & 17.31981617 & 2.66648815 \\
\hline $\mathrm{C}$ & 10.50880398 & 6.94113862 & -1.91070449 \\
\hline $\mathrm{H}$ & 11.53432104 & 6.82709320 & -1.62749361 \\
\hline $\mathrm{H}$ & 9.97870302 & 7.45083736 & -1.13346568 \\
\hline $\mathrm{H}$ & 10.07251853 & 5.97667534 & -2.06679926 \\
\hline $\mathrm{C}$ & 10.06121867 & 11.28194284 & -4.45606947 \\
\hline $\mathrm{H}$ & 9.27670461 & 11.59334438 & -3.79844741 \\
\hline $\mathrm{H}$ & 10.98467482 & 11.72293567 & -4.14357298 \\
\hline $\mathrm{H}$ & 9.83578632 & 11.59568687 & -5.45388956 \\
\hline $\mathrm{H}$ & 9.73404115 & 10.63336701 & -1.72064939 \\
\hline
\end{tabular}


SCF done: -1805.79951838 Hartree

No imaginary Frequency.

Compound $\mathbf{2 b}$, optimized $\mathrm{S}_{0}$ state Geometry.

$\begin{array}{lrrr}\mathrm{B} & 10.67910000 & 11.55710000 & 0.67190000 \\ \mathrm{C} & 11.54620000 & 9.83010000 & -1.11990000 \\ \mathrm{C} & 12.74680000 & 9.00550000 & -1.24960000 \\ \mathrm{H} & 12.94380000 & 8.44600000 & -1.96570000 \\ \mathrm{C} & 13.50890000 & 9.18770000 & -0.17540000 \\ \mathrm{H} & 14.33580000 & 8.79220000 & -0.01620000 \\ \mathrm{C} & 12.82450000 & 10.11120000 & 0.69700000 \\ \mathrm{C} & 13.22750000 & 10.55590000 & 1.94660000 \\ \mathrm{C} & 12.42970000 & 11.46230000 & 2.60230000 \\ \mathrm{C} & 12.57730000 & 12.01770000 & 3.92700000 \\ \mathrm{H} & 13.28240000 & 11.85810000 & 4.51200000 \\ \mathrm{C} & 11.52120000 & 12.80060000 & 4.16420000 \\ \mathrm{H} & 11.37520000 & 13.27720000 & 4.94820000 \\ \mathrm{C} & 10.64200000 & 12.79530000 & 3.01780000 \\ \mathrm{C} & 10.50800000 & 9.93430000 & -1.95980000 \\ \mathrm{H} & 9.83550000 & 10.54190000 & -1.75060000 \\ \mathrm{C} & 10.37070000 & 9.14210000 & -3.19320000 \\ \mathrm{C} & 10.18570000 & 9.74750000 & -4.41620000 \\ \mathrm{C} & 10.08640000 & 9.06790000 & -5.59210000 \\ \mathrm{H} & 9.96950000 & 9.52510000 & -6.39220000 \\ \mathrm{C} & 10.16160000 & 7.69840000 & -5.58030000 \\ \mathrm{H} & 10.10940000 & 7.22030000 & -6.37600000 \\ \mathrm{C} & 10.31370000 & 7.03120000 & -4.38080000 \\ \mathrm{H} & 10.34270000 & 6.10060000 & -4.36020000 \\ \mathrm{C} & 10.42370000 & 7.75980000 & -3.21230000 \\ \mathrm{C} & 14.42620000 & 9.99950000 & 2.64350000 \\ \mathrm{C} & 14.43570000 & 8.73600000 & 3.16810000 \\ \mathrm{C} & 15.45440000 & 8.24010000 & 3.96380000 \\ \mathrm{C} & 15.40980000 & 7.38560000 & 4.32780000 \\ \mathrm{C} & 16.53160000 & 9.05420000 & 4.19370000 \\ \mathrm{C} & 17.20110000 & 8.75790000 & 4.76690000 \\ \mathrm{C} & 16.66300000 & 10.27990000 & 3.62050000 \\ \mathrm{C} & 17.43440000 & 10.78520000 & 3.74130000 \\ \mathrm{C} & 15.59480000 & 10.75860000 & 2.83800000 \\ \mathrm{C} & 9.47690000 & 13.40690000 & 2.88670000 \\ \mathrm{C} & 9.01810000 & 13.33640000 & 2.08060000 \\ \mathrm{C} & & & \\ \mathrm{H} & 8.48540000 & 14.20220000 & 3.98300000 \\ \mathrm{C} & 13.62870000 & 5.16030000\end{array}$




$\begin{array}{lrrr}\mathrm{C} & 7.90450000 & 14.38060000 & 6.20510000 \\ \mathrm{H} & 7.59630000 & 13.97580000 & 6.98310000 \\ \mathrm{C} & 7.86500000 & 15.75540000 & 6.05330000 \\ \mathrm{H} & 7.54380000 & 16.27750000 & 6.75180000 \\ \mathrm{C} & 8.28090000 & 16.36020000 & 4.91720000 \\ \mathrm{H} & 8.22400000 & 17.28490000 & 4.82440000 \\ \mathrm{C} & 8.79070000 & 15.58110000 & 3.89900000 \\ \mathrm{Cl} & 10.04520000 & 11.47940000 & -4.46120000 \\ \mathrm{Cl} & 10.51990000 & 6.83440000 & -1.74100000 \\ \mathrm{Cl} & 13.08380000 & 7.67720000 & 2.88510000 \\ \mathrm{Cl} & 15.70760000 & 12.29660000 & 2.05770000 \\ \mathrm{Cl} & 9.35770000 & 16.36770000 & 2.45080000 \\ \mathrm{Cl} & 8.41840000 & 11.91170000 & 5.34530000 \\ \mathrm{~F} & 9.44390000 & 10.98380000 & 0.82270000 \\ \mathrm{~F} & 10.57150000 & 12.65060000 & -0.13600000 \\ \mathrm{~N} & 11.67900000 & 10.52500000 & 0.10170000 \\ \mathrm{~N} & 11.25810000 & 11.93790000 & 2.06740000\end{array}$

SCF done: -4209.50166954 Hartree

No imaginary Frequency.

Compound 7, optimized $\mathrm{S}_{0}$ state Geometry.

\begin{tabular}{|c|c|c|c|}
\hline B & 10.67910000 & 11.55710000 & 0.67190000 \\
\hline $\mathrm{C}$ & 11.54620000 & 9.83010000 & -1.11990000 \\
\hline $\mathrm{C}$ & 12.74680000 & 9.00550000 & -1.24960000 \\
\hline $\mathrm{H}$ & 12.94380000 & 8.44600000 & -1.96570000 \\
\hline $\mathrm{C}$ & 13.50890000 & 9.18770000 & -0.17540000 \\
\hline $\mathrm{H}$ & 14.33580000 & 8.79220000 & -0.01620000 \\
\hline $\mathrm{C}$ & 12.82450000 & 10.11120000 & 0.69700000 \\
\hline $\mathrm{C}$ & 13.22750000 & 10.55590000 & 1.94660000 \\
\hline $\mathrm{C}$ & 12.42970000 & 11.46230000 & 2.60230000 \\
\hline $\mathrm{C}$ & 12.57730000 & 12.01770000 & 3.92700000 \\
\hline $\mathrm{H}$ & 13.28240000 & 11.85810000 & 4.51200000 \\
\hline $\mathrm{C}$ & 11.52120000 & 12.80060000 & 4.16420000 \\
\hline $\mathrm{H}$ & 11.37520000 & 13.27720000 & 4.94820000 \\
\hline $\mathrm{C}$ & 10.64200000 & 12.79530000 & 3.01780000 \\
\hline $\mathrm{C}$ & 10.50800000 & 9.93430000 & -1.95980000 \\
\hline $\mathrm{H}$ & 10.46378841 & 10.96143515 & -2.25634463 \\
\hline $\mathrm{C}$ & 10.37070000 & 9.14210000 & -3.19320000 \\
\hline $\mathrm{C}$ & 10.18570000 & 9.74750000 & -4.41620000 \\
\hline $\mathrm{C}$ & 10.08640000 & 9.06790000 & -5.59210000 \\
\hline $\mathrm{H}$ & 9.96950000 & 9.52510000 & -6.39220000 \\
\hline $\mathrm{C}$ & 10.16160000 & 7.69840000 & -5.58030000 \\
\hline $\mathrm{H}$ & 10.10940000 & 7.22030000 & -6.37600000 \\
\hline
\end{tabular}




$\begin{array}{lrrr}\mathrm{C} & 10.31370000 & 7.03120000 & -4.38080000 \\ \mathrm{H} & 10.34270000 & 6.10060000 & -4.36020000 \\ \mathrm{C} & 10.42370000 & 7.75980000 & -3.21230000 \\ \mathrm{C} & 14.42620000 & 9.99950000 & 2.64350000 \\ \mathrm{C} & 14.43570000 & 8.73600000 & 3.16810000 \\ \mathrm{C} & 15.45440000 & 8.24010000 & 3.96380000 \\ \mathrm{H} & 15.40980000 & 7.38560000 & 4.32780000 \\ \mathrm{C} & 16.53160000 & 9.05420000 & 4.19370000 \\ \mathrm{H} & 17.20110000 & 8.75790000 & 4.76690000 \\ \mathrm{C} & 16.66300000 & 10.27990000 & 3.62050000 \\ \mathrm{H} & 17.43440000 & 10.78520000 & 3.74130000 \\ \mathrm{C} & 15.59480000 & 10.75860000 & 2.83800000 \\ \mathrm{C} & 9.47690000 & 13.40690000 & 2.88670000 \\ \mathrm{H} & 8.76071842 & 12.65429712 & 2.63062803 \\ \mathrm{C} & 8.88540000 & 14.20220000 & 3.98300000 \\ \mathrm{C} & 8.41570000 & 13.62870000 & 5.16030000 \\ \mathrm{C} & 7.90450000 & 14.38060000 & 6.20510000 \\ \mathrm{H} & 7.59630000 & 13.97580000 & 6.98310000 \\ \mathrm{C} & 7.86500000 & 15.75540000 & 6.05330000 \\ \mathrm{H} & 7.54380000 & 16.27750000 & 6.75180000 \\ \mathrm{C} & 8.28090000 & 16.36020000 & 4.91720000 \\ \mathrm{H} & 8.22400000 & 17.28490000 & 4.82440000 \\ \mathrm{C} & 8.79070000 & 15.58110000 & 3.89900000 \\ \mathrm{Cl} & 10.04520000 & 11.47940000 & -4.46120000 \\ \mathrm{Cl} & 10.51990000 & 6.83440000 & -1.74100000 \\ \mathrm{Cl} & 13.08380000 & 7.67720000 & 2.88510000 \\ \mathrm{Cl} & 15.70760000 & 12.29660000 & 2.05770000 \\ \mathrm{Cl} & 9.35770000 & 16.36770000 & 2.45080000 \\ \mathrm{Cl} & 8.41840000 & 11.91170000 & 5.34530000 \\ \mathrm{~F} & 9.44390000 & 10.98380000 & 0.82270000 \\ \mathrm{~F} & 10.57150000 & 12.65060000 & -0.13600000 \\ \mathrm{~N} & 11.67900000 & 10.52500000 & 0.10170000 \\ \mathrm{H} & 11.25810000 & 11.93790000 & 2.06740000 \\ \mathrm{SCF} & 9.56497254 & 14.06071745 & 2.04428326 \\ \mathrm{H} & 9.73920104 & -1.37861604\end{array}$

SCF done: -4210.74336205 Hartree

No imaginary Frequency.

Compound 8, optimized $\mathrm{S}_{0}$ state Geometry.

$\begin{array}{lrrr}\text { B } & -3.11783200 & 0.00333200 & 0.02600800 \\ \text { C } & -2.55387500 & 2.53514800 & -0.03174400 \\ \text { C } & -1.42551200 & 3.37944400 & -0.03878000 \\ \text { C } & -0.30836500 & 2.55296400 & -0.02331100\end{array}$




$\begin{array}{lrrr}\mathrm{C} & -0.77791800 & 1.21373400 & -0.00801900 \\ \mathrm{C} & -0.07600800 & -0.00014900 & -0.00275200 \\ \mathrm{C} & -0.78068300 & -1.21244500 & -0.00751600 \\ \mathrm{C} & -0.31424200 & -2.55276600 & -0.02217900 \\ \mathrm{C} & -1.43330200 & -3.37666300 & -0.03731200 \\ \mathrm{C} & -2.55970600 & -2.52975800 & -0.03068200 \\ \mathrm{C} & 1.42032700 & -0.00159800 & 0.00137600 \\ \mathrm{C} & 2.11518600 & -0.00411600 & 1.22923700 \\ \mathrm{C} & 3.51373100 & -0.00908300 & 1.20748100 \\ \mathrm{C} & 4.23693800 & -0.00870100 & 0.01084300 \\ \mathrm{C} & 3.52176900 & -0.01027100 & -1.19059700 \\ \mathrm{C} & 2.12335400 & -0.00530800 & -1.22182800 \\ \mathrm{~F} & -3.86242500 & 0.00440400 & 1.20147400 \\ \mathrm{~F} & -3.96064500 & 0.00410200 & -1.07982800 \\ \mathrm{~N} & -2.17423900 & 1.24622000 & -0.01307300 \\ \mathrm{~N} & -2.17708000 & -1.24170100 & -0.01259600 \\ \mathrm{H} & -1.44656400 & 4.46029700 & -0.05487600 \\ \mathrm{H} & 0.73219300 & 2.84693200 & -0.02545600 \\ \mathrm{H} & 0.72562000 & -2.84922200 & -0.02414100 \\ \mathrm{H} & -1.45682700 & -4.45747300 & -0.05292100 \\ \mathrm{H} & -3.60876500 & -2.79506100 & -0.03793600 \\ \mathrm{H} & 4.05068800 & -0.01471900 & 2.15372700 \\ \mathrm{H} & 4.06509300 & -0.01686100 & -2.13319600 \\ \mathrm{H} & -3.60232500 & 2.80287300 & -0.03909900 \\ \mathrm{H} & 5.30693102 & -0.00724892 & 0.01442514 \\ \mathrm{Cl} & 1.26140379 & -0.00589847 & 2.76827981 \\ \mathrm{Cl} & 1.28039985 & -0.00845054 & -2.76682587 \\ \mathrm{SCF} & & \end{array}$

SCF done: -1831.62251886 Hartree

No imaginary Frequency. 


\section{Cytotoxicity of $2 \mathrm{~b}$}

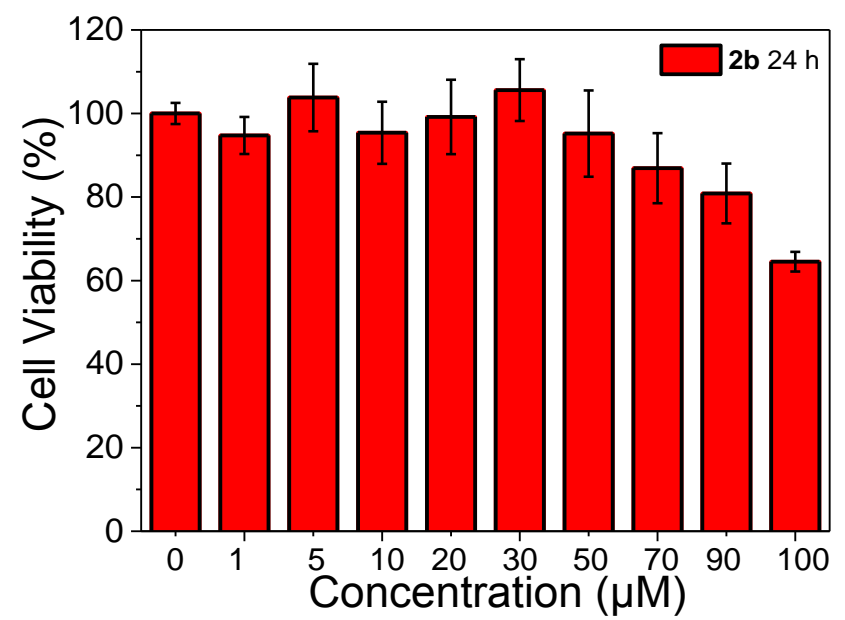

Figure S17. Cytotoxicity of HeLa cells treated with different concentrations of $\mathbf{2 b}$ for $24 \mathrm{~h}$ as demonstrated by CCK-8 assay. 
7. Copies of ${ }^{1} \mathrm{H}$ and ${ }^{13} \mathrm{C}$ NMR spectra for all new compounds

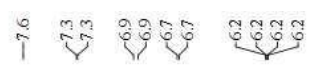
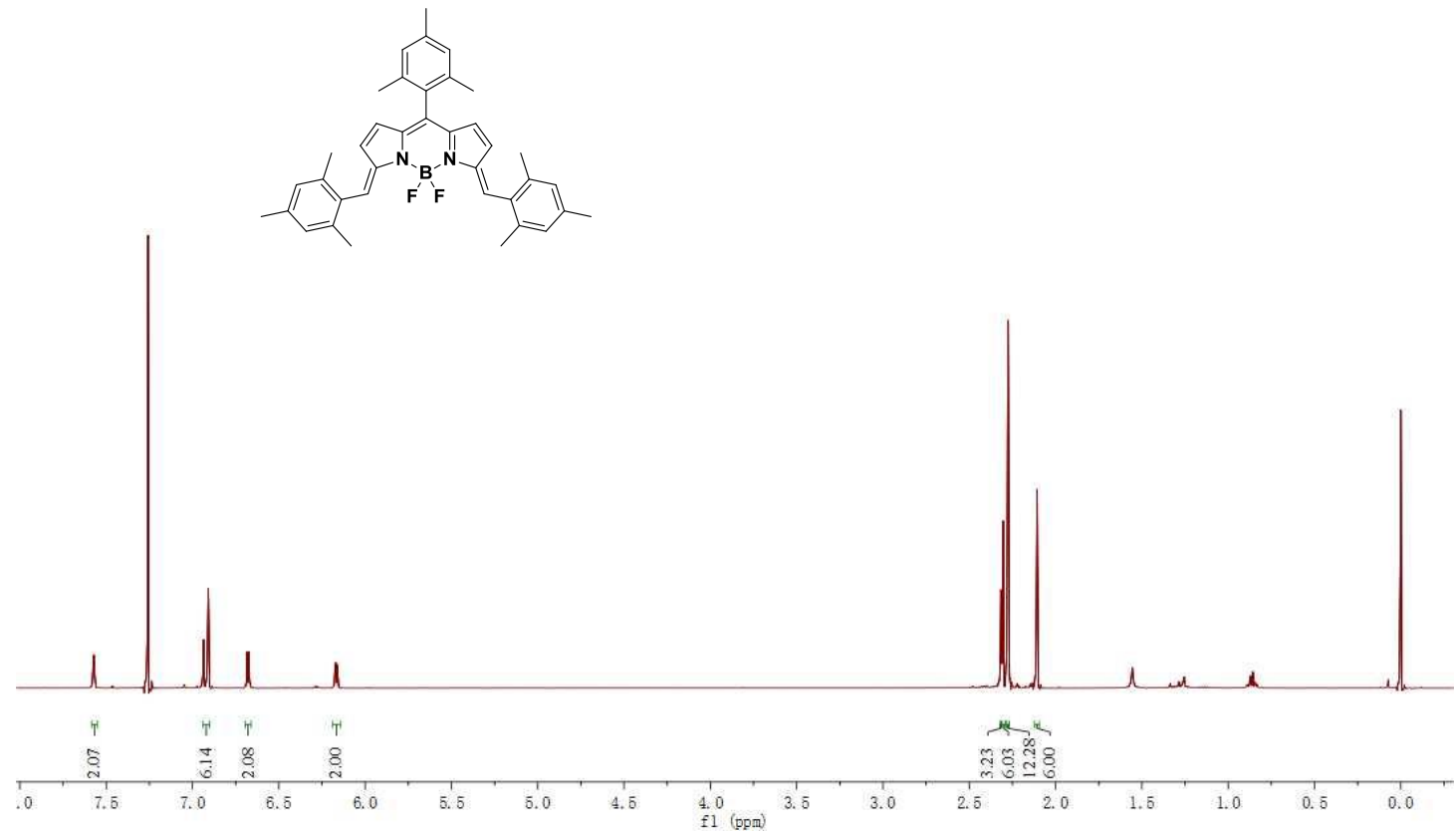

${ }^{1} \mathrm{H}$ NMR spectrum of $\mathbf{2 a}\left(\mathrm{CDCl}_{3}, 500 \mathrm{MHz}\right)$

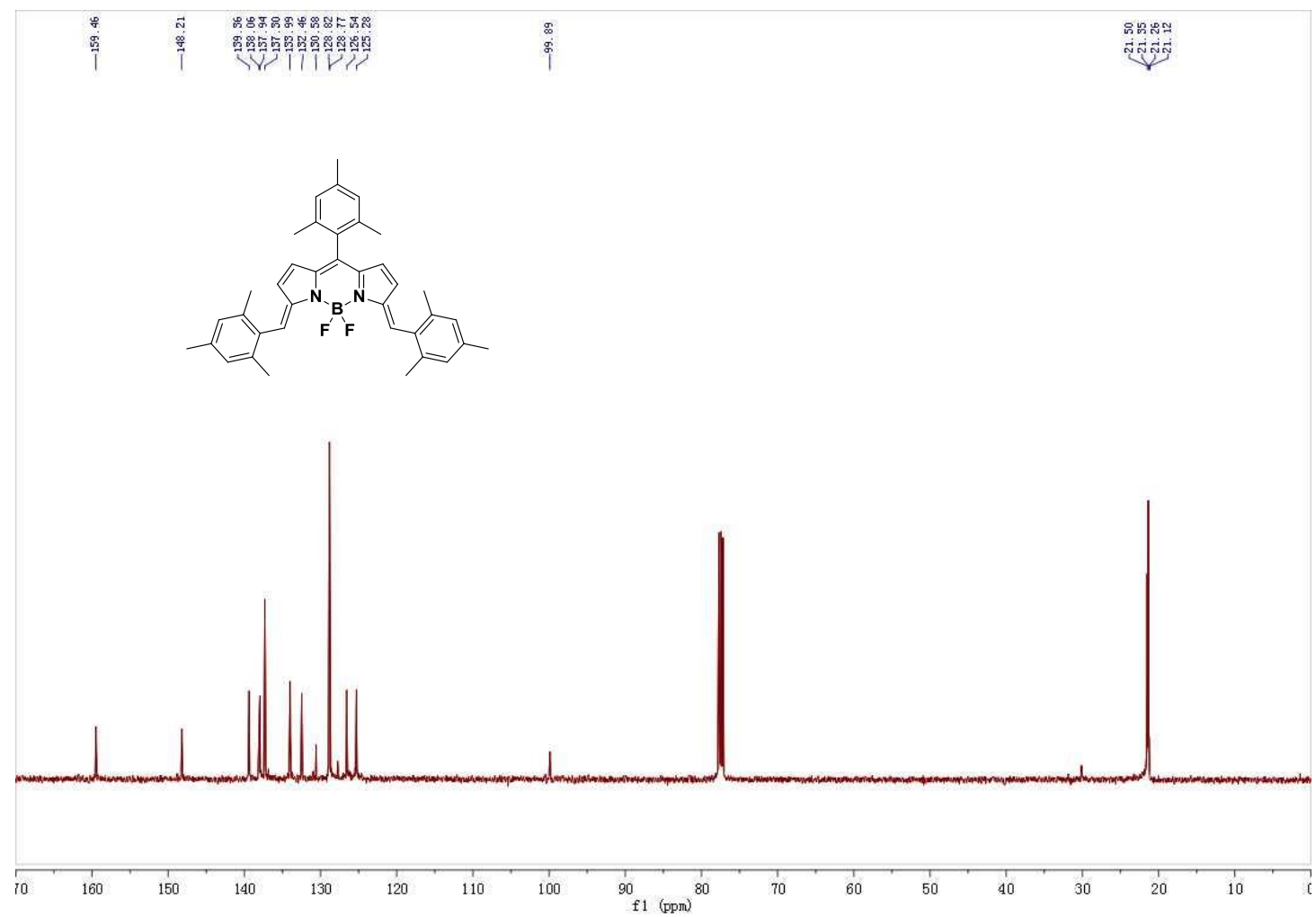

${ }^{13} \mathrm{C}\left\{{ }^{1} \mathrm{H}\right\} \mathrm{NMR}$ spectrum of $\mathbf{2 a}\left(\mathrm{CDCl}_{3}, 126 \mathrm{MHz}\right)$ 

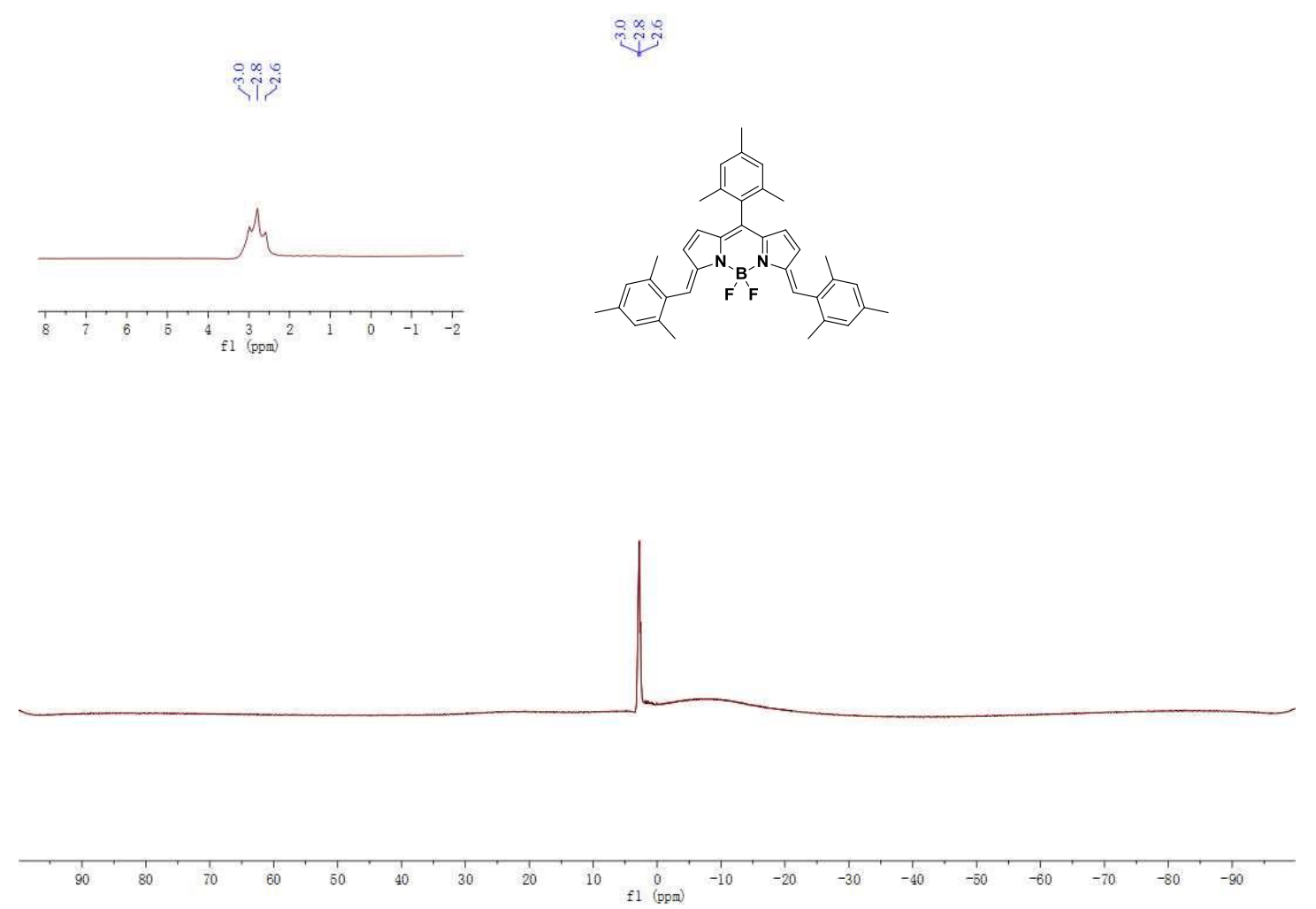

${ }^{11} \mathrm{~B}$ NMR spectrum of $\mathbf{2 a}\left(\mathrm{CDCl}_{3}, 160 \mathrm{MHz}\right)$

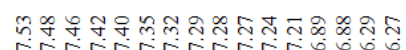

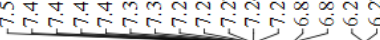
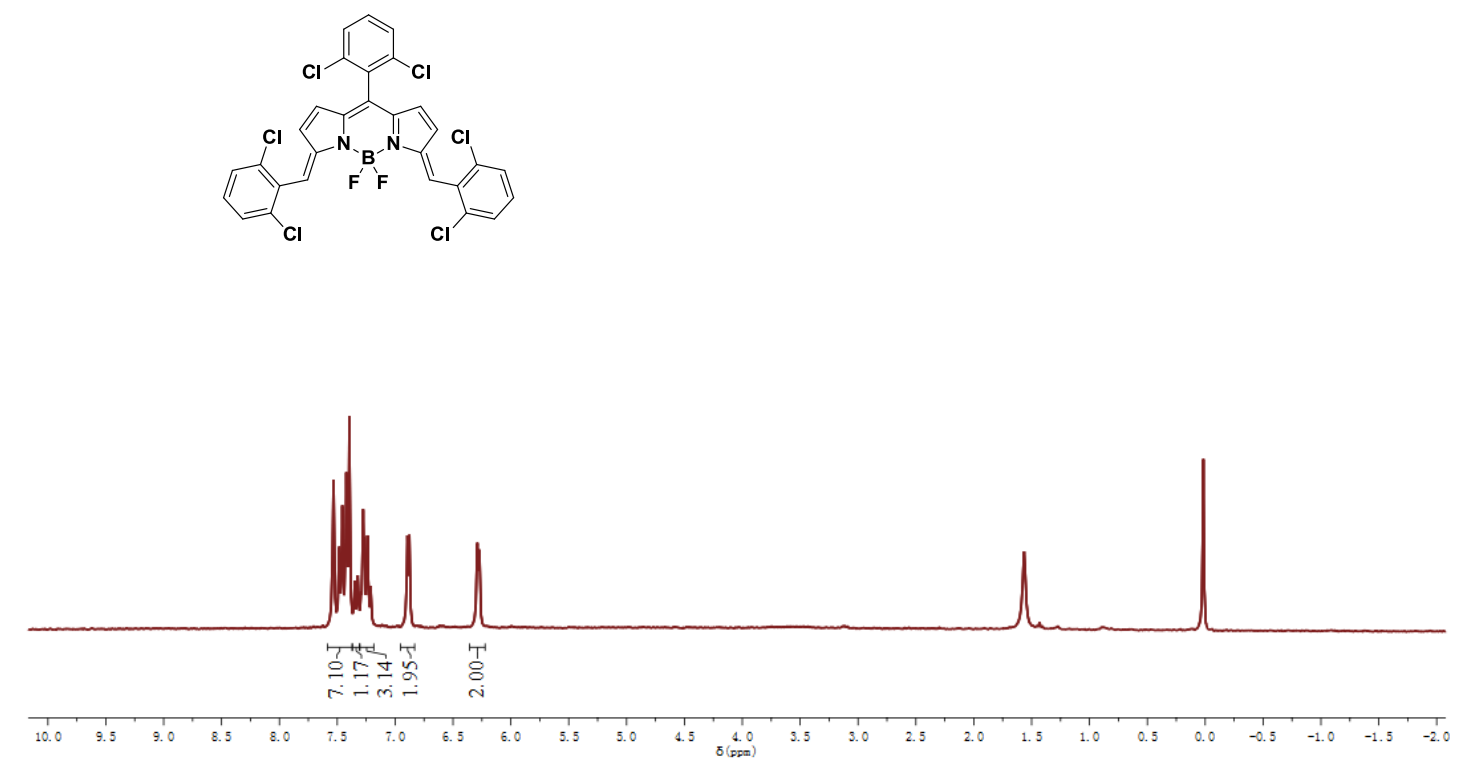

${ }^{1} \mathrm{H}$ NMR spectrum of $\mathbf{2 b}\left(\mathrm{CDCl}_{3}, 300 \mathrm{MHz}\right)$ 


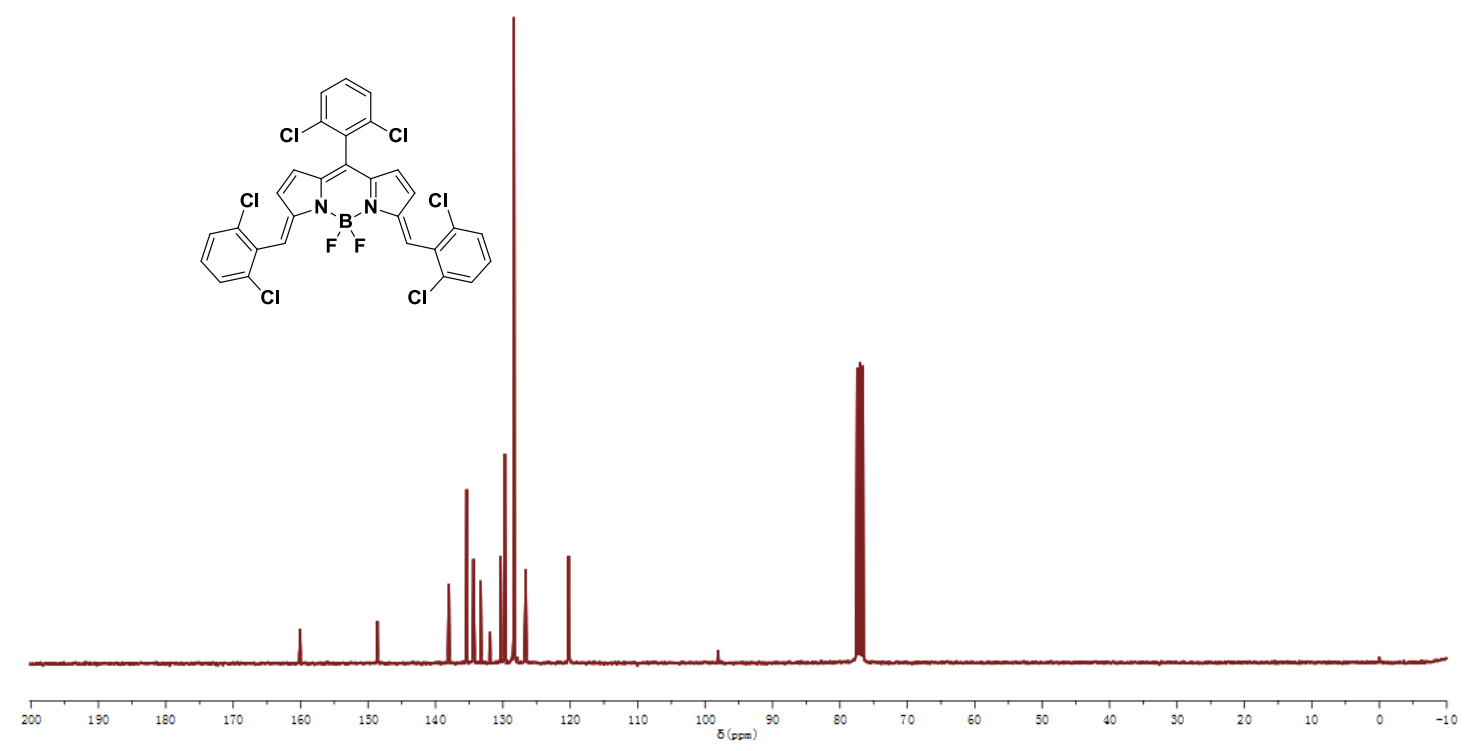

${ }^{13} \mathrm{C}\left\{{ }^{1} \mathrm{H}\right\}$ NMR spectrum of $\mathbf{2 b}\left(\mathrm{CDCl}_{3}, 126 \mathrm{MHz}\right)$
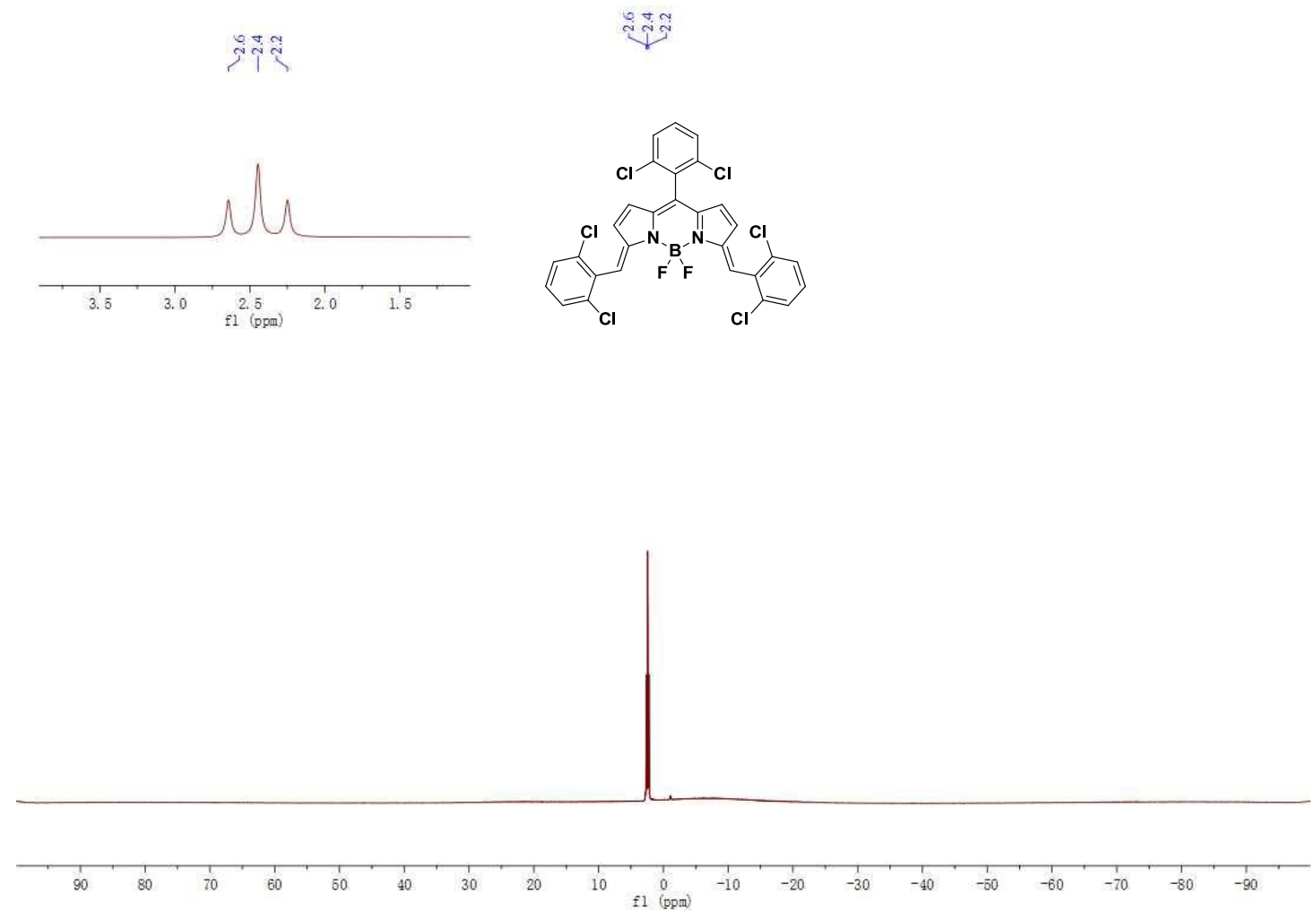

${ }^{11} \mathrm{~B}$ NMR spectrum of $\mathbf{2 b}\left(\mathrm{CDCl}_{3}, 160 \mathrm{MHz}\right)$ 


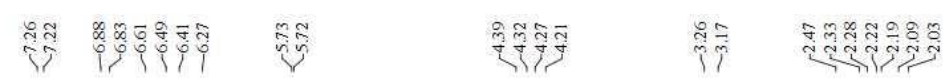

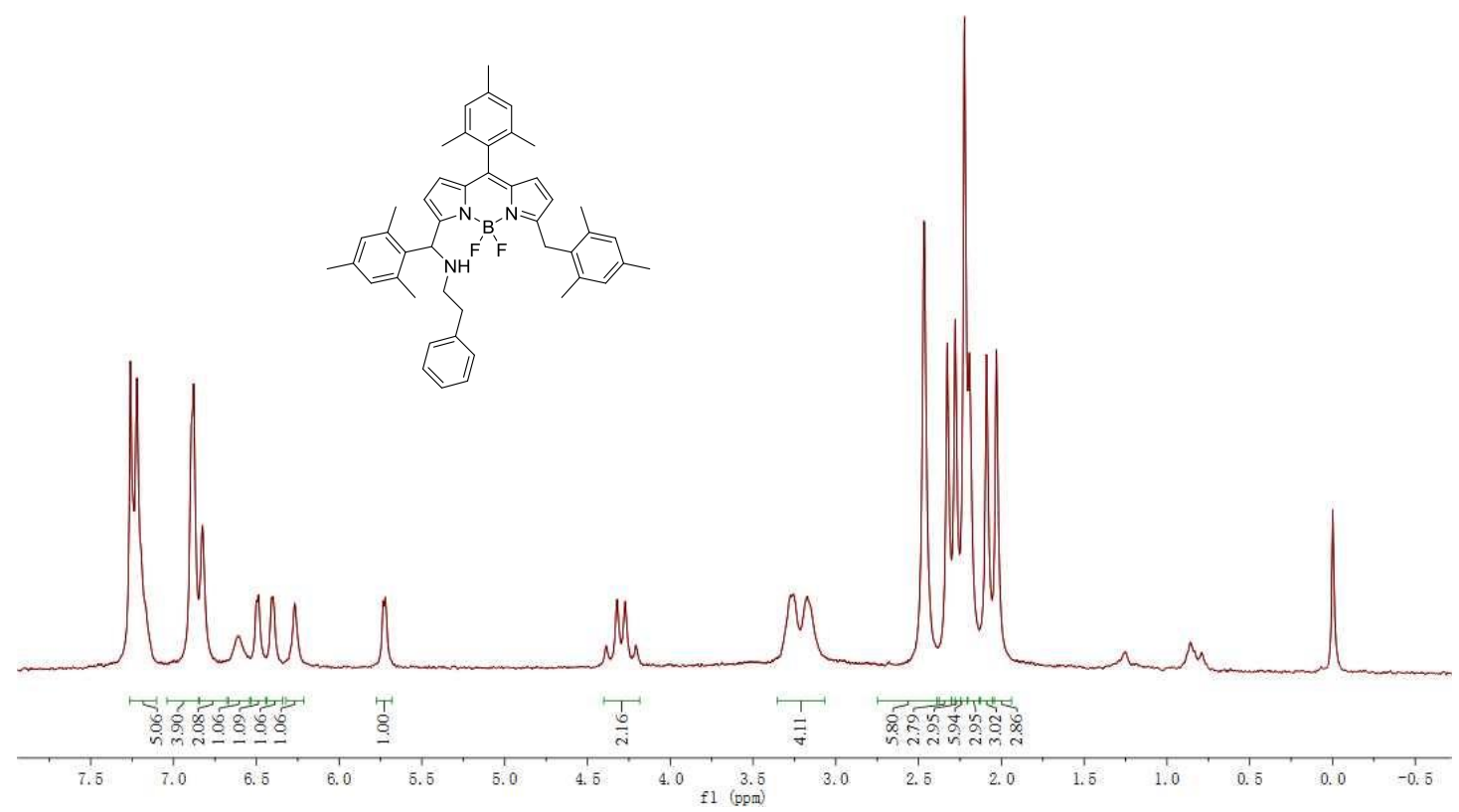

${ }^{1} \mathrm{H}$ NMR spectrum of $\mathbf{3 a}\left(\mathrm{CDCl}_{3}, 300 \mathrm{MHz}\right)$

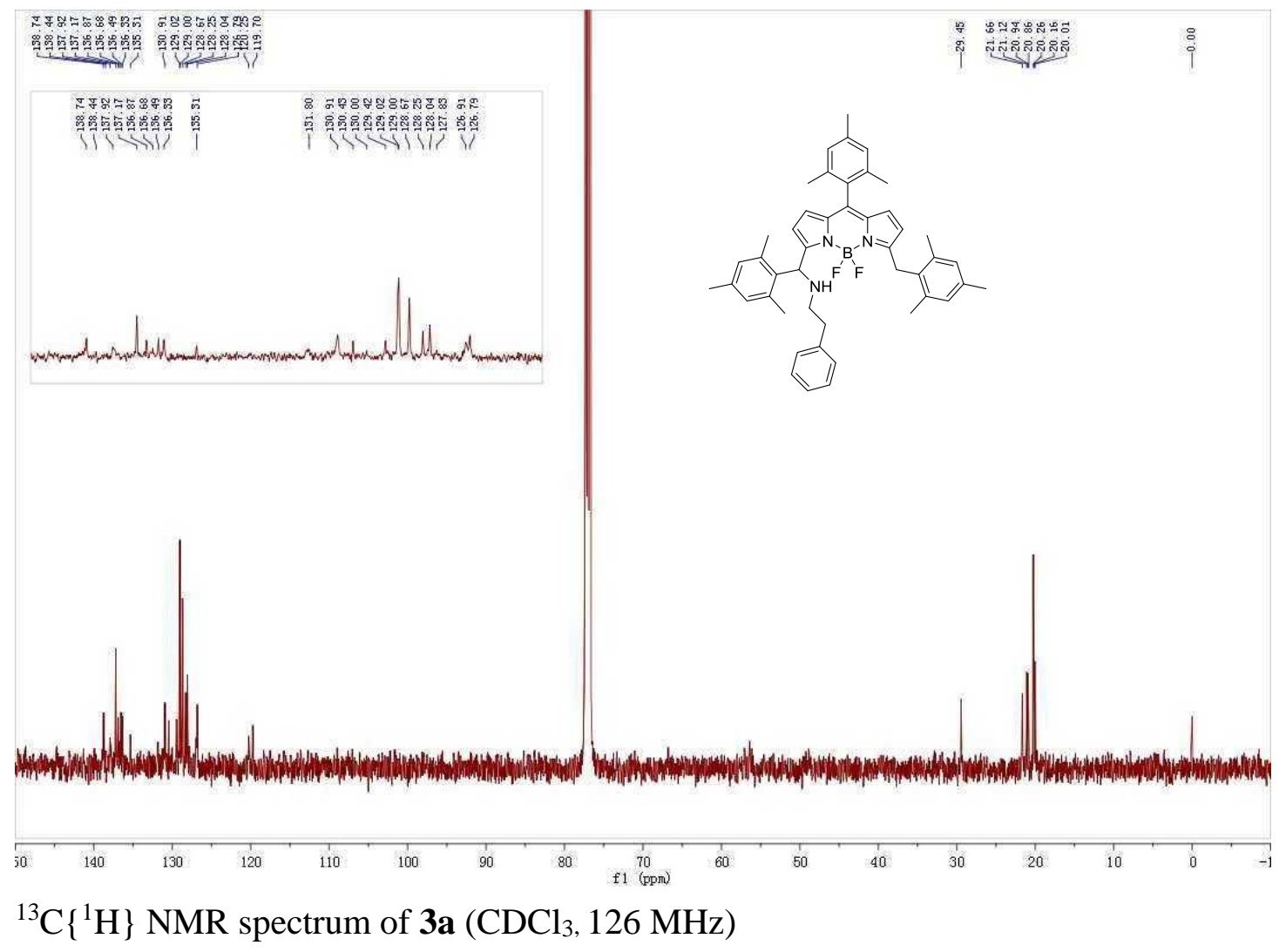




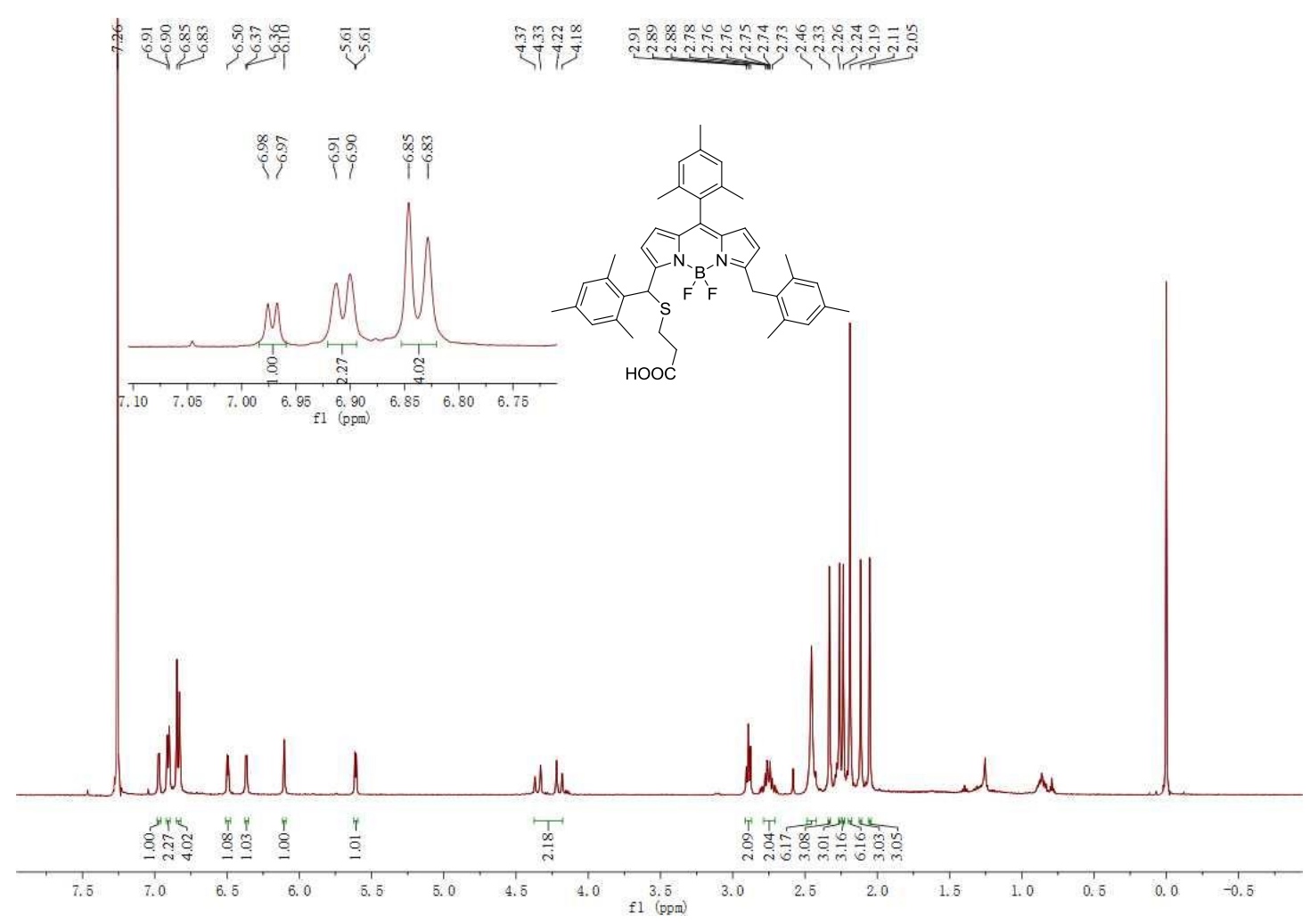

${ }^{1} \mathrm{H}$ NMR spectrum of $\mathbf{4 a}\left(\mathrm{CDCl}_{3}, 500 \mathrm{MHz}\right)$

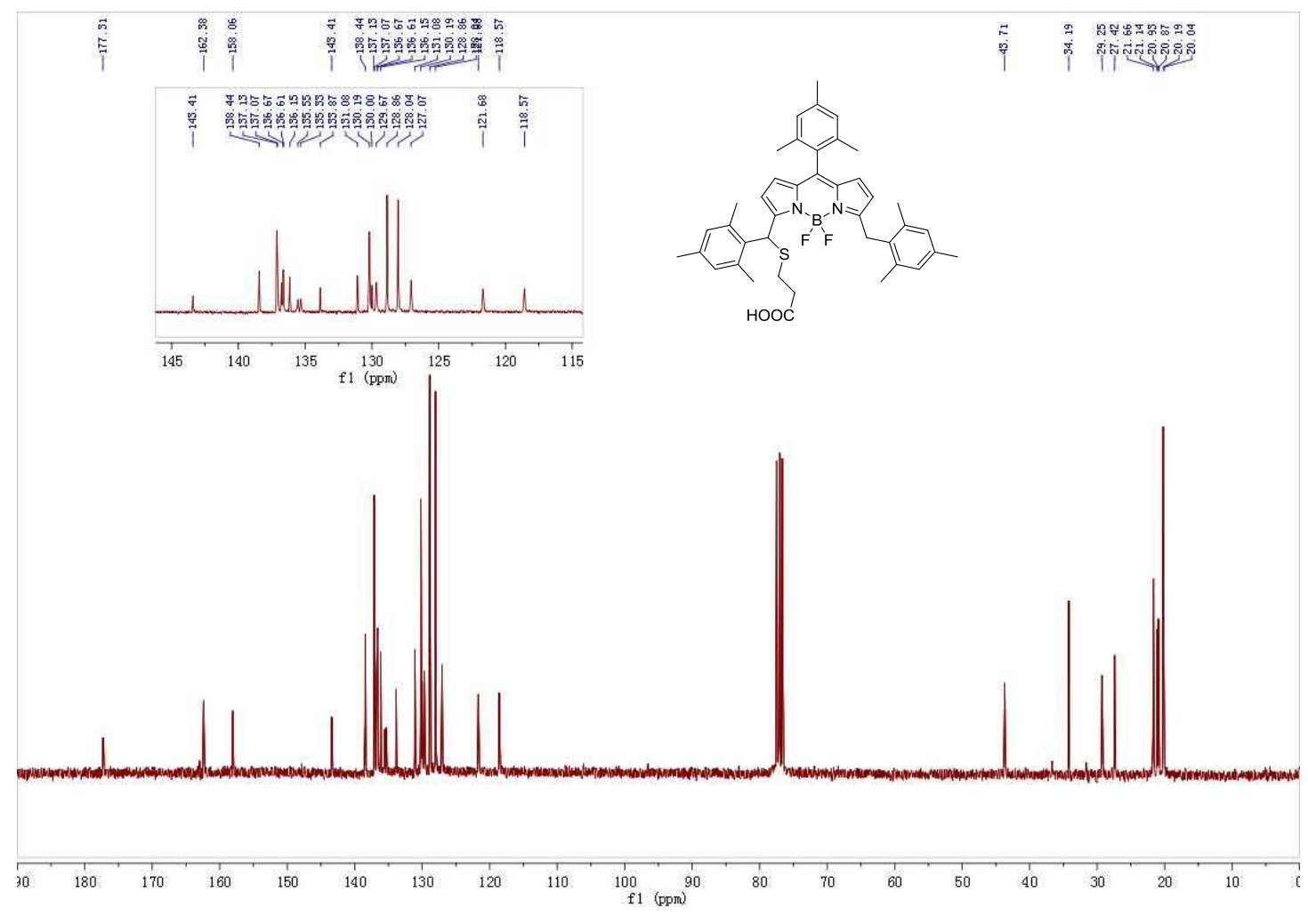

${ }^{13} \mathrm{C}\left\{{ }^{1} \mathrm{H}\right\}$ NMR spectrum of $\mathbf{4 a}\left(\mathrm{CDCl}_{3}, 75 \mathrm{MHz}\right)$ 

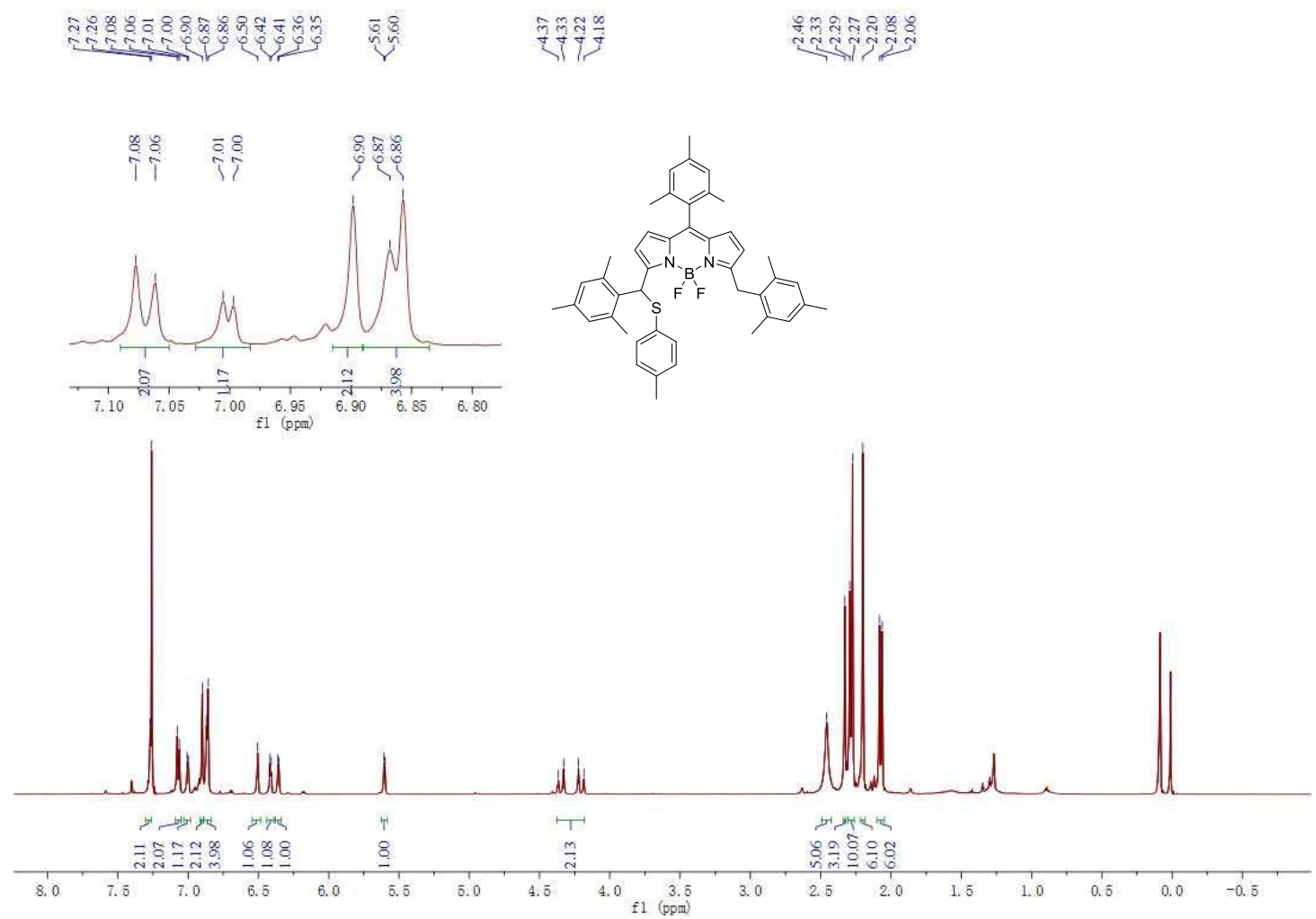

${ }^{1} \mathrm{H}$ NMR spectrum of $\mathbf{5 a}\left(\mathrm{CDCl}_{3}, 500 \mathrm{MHz}\right)$

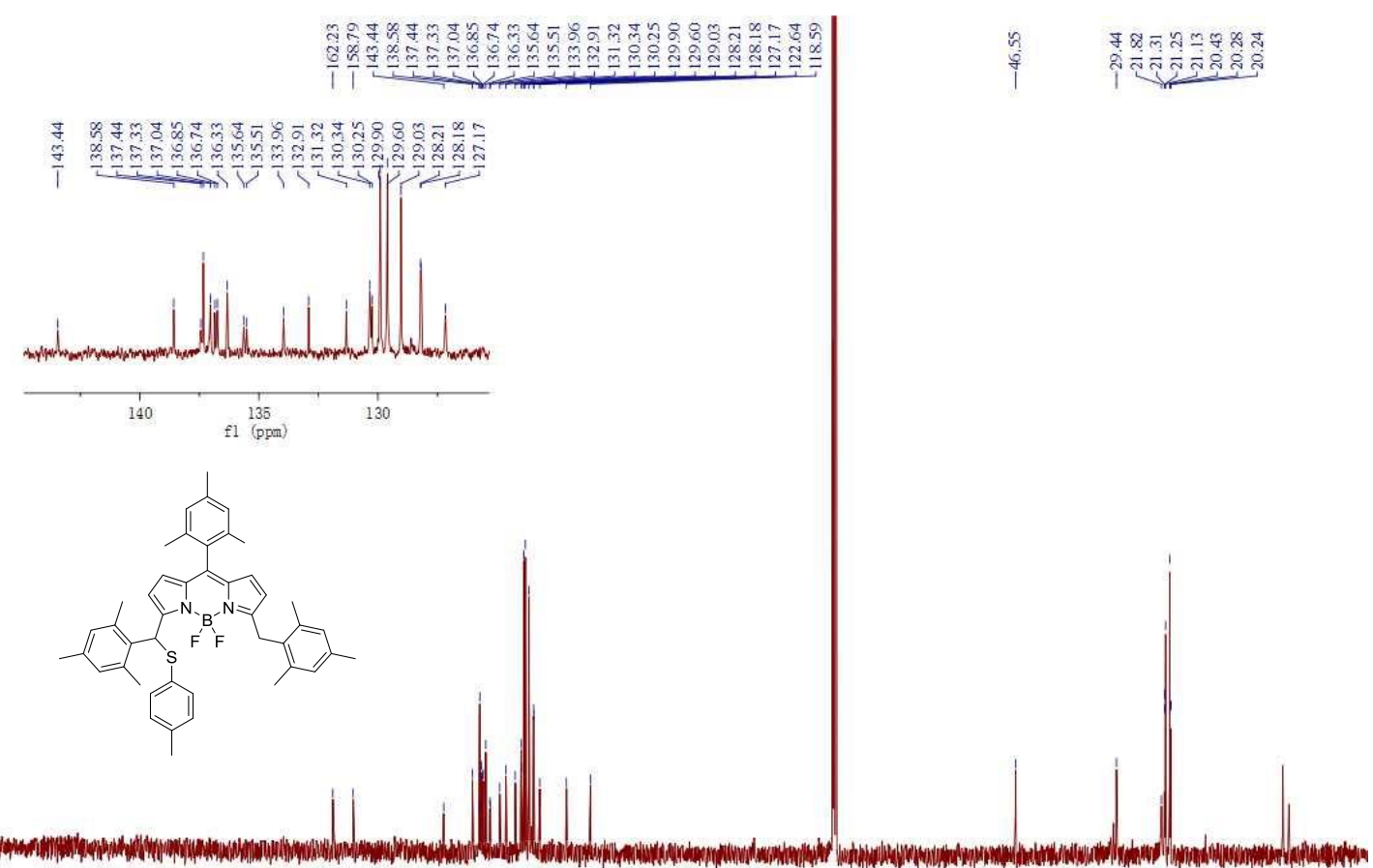

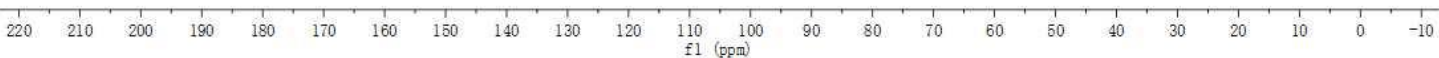

${ }^{13} \mathrm{C}\left\{{ }^{1} \mathrm{H}\right\}$ NMR spectrum of $\mathbf{5 a}\left(\mathrm{CDCl}_{3}, 126 \mathrm{MHz}\right)$ 

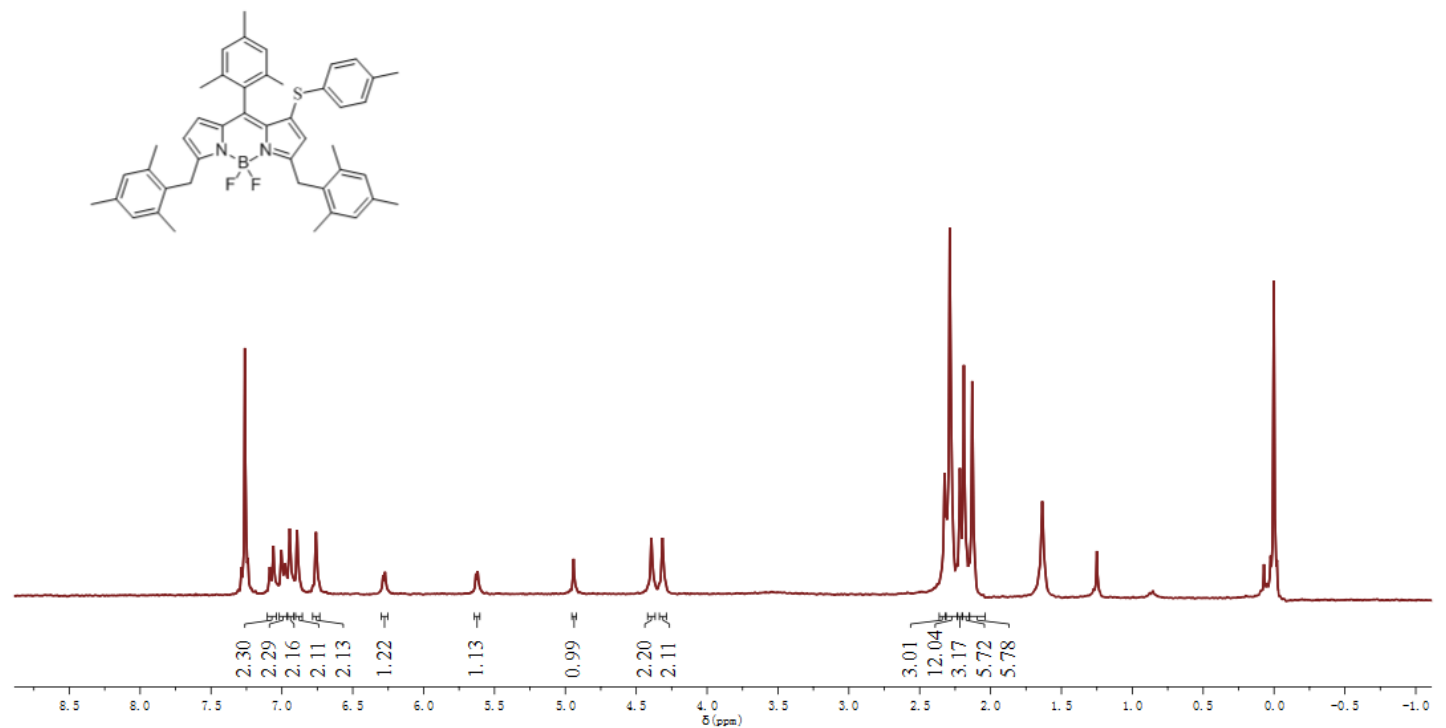

${ }^{1} \mathrm{H}$ NMR spectrum of $\mathbf{6 a}\left(\mathrm{CDCl}_{3}, 300 \mathrm{MHz}\right)$
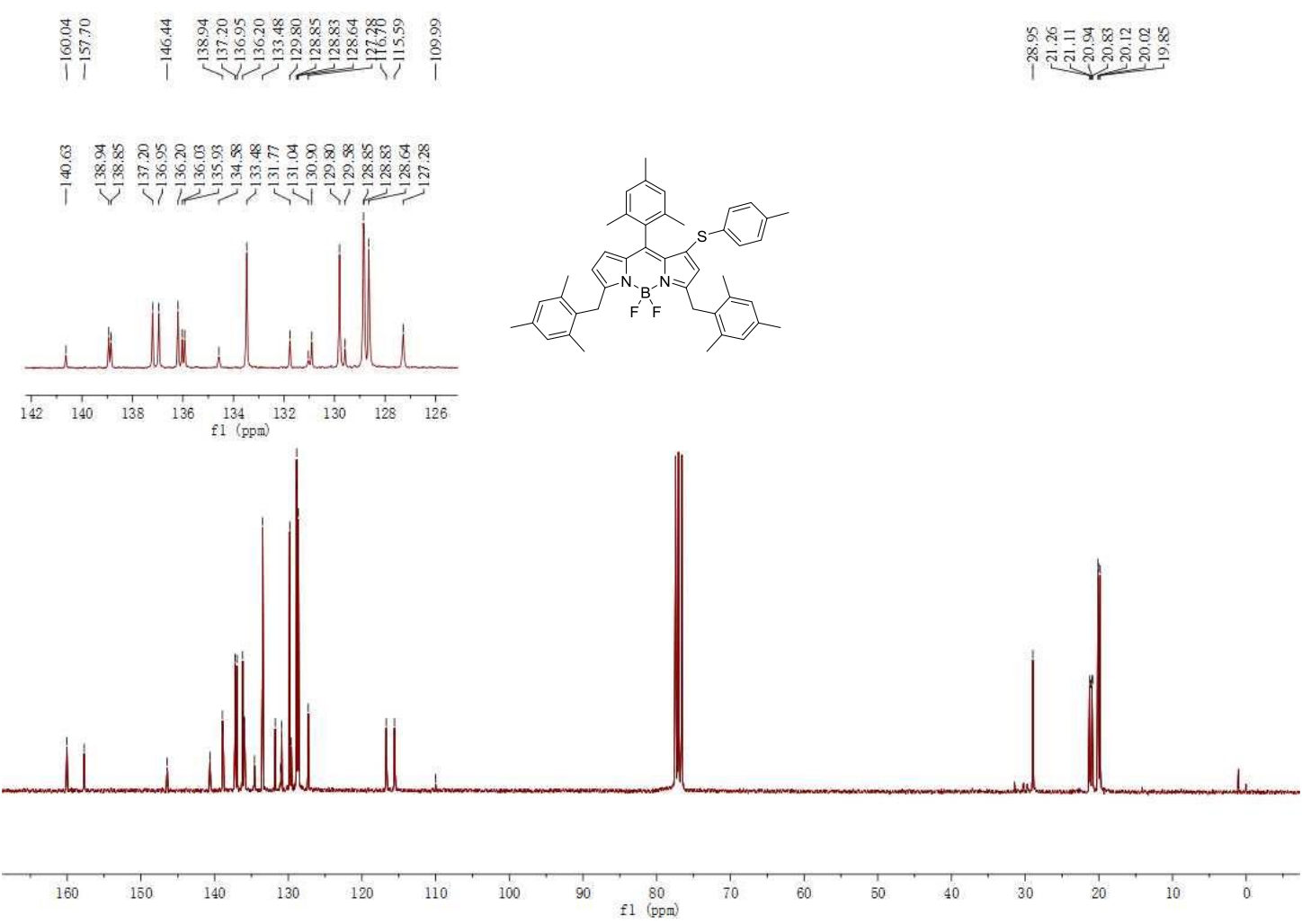

${ }^{13} \mathrm{C}\left\{{ }^{1} \mathrm{H}\right\}$ NMR spectrum of $\mathbf{6 a}\left(\mathrm{CDCl}_{3}, 75 \mathrm{MHz}\right)$ 

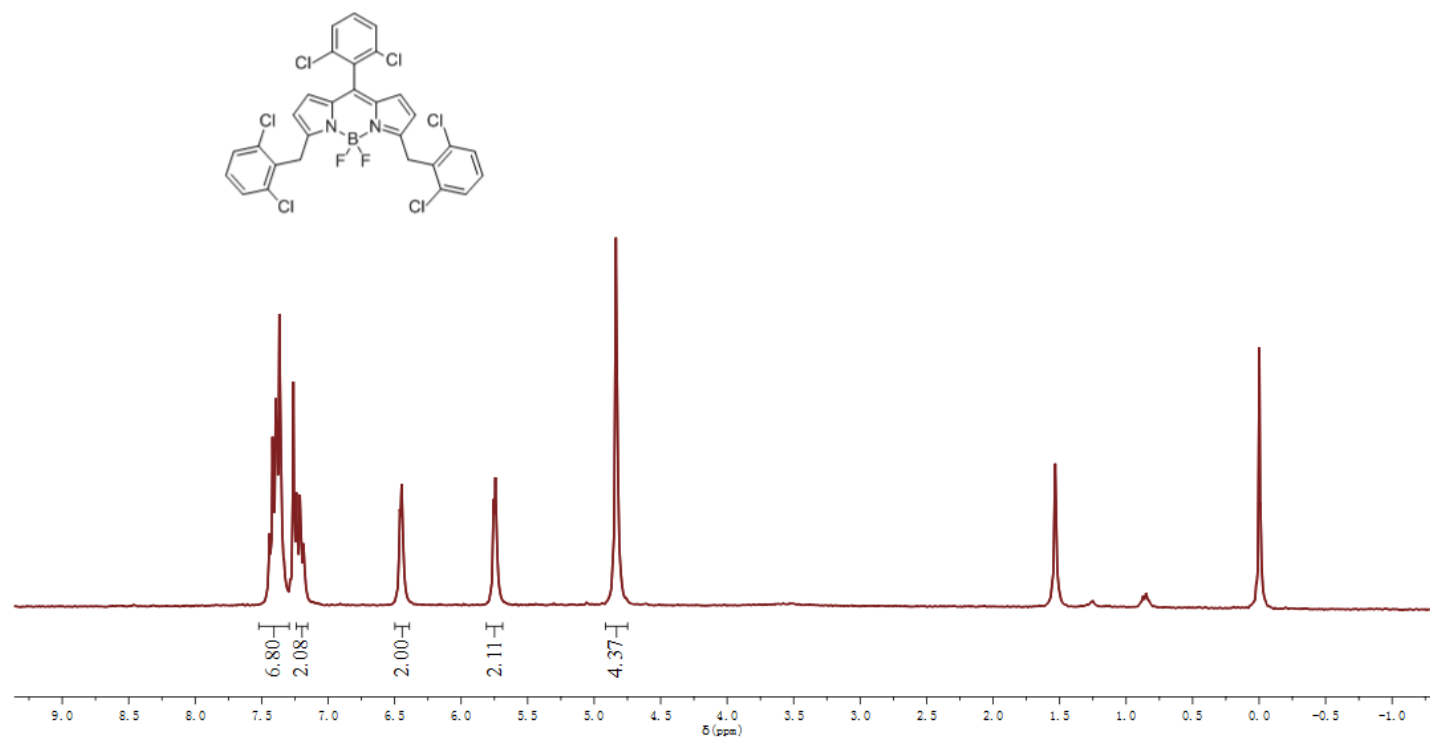

${ }^{1} \mathrm{H} \mathrm{NMR}$ spectrum of $7\left(\mathrm{CDCl}_{3}, 300 \mathrm{MHz}\right)$
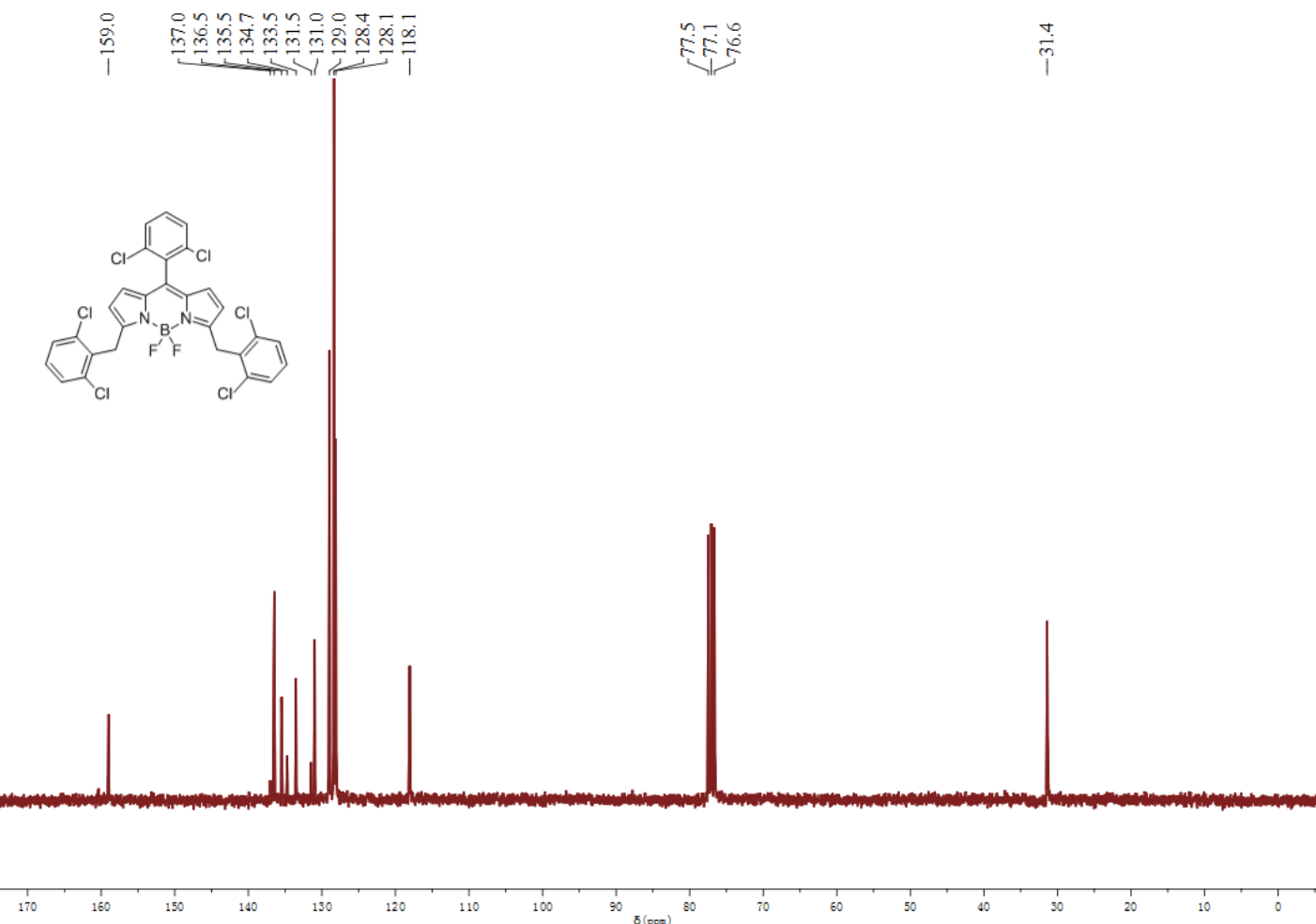

${ }^{13} \mathrm{C}\left\{{ }^{1} \mathrm{H}\right\}$ NMR spectrum of $7\left(\mathrm{CDCl}_{3}, 75 \mathrm{MHz}\right)$ 


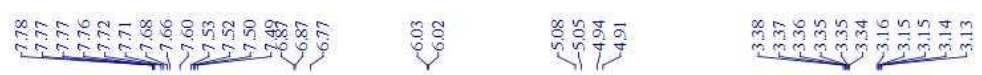

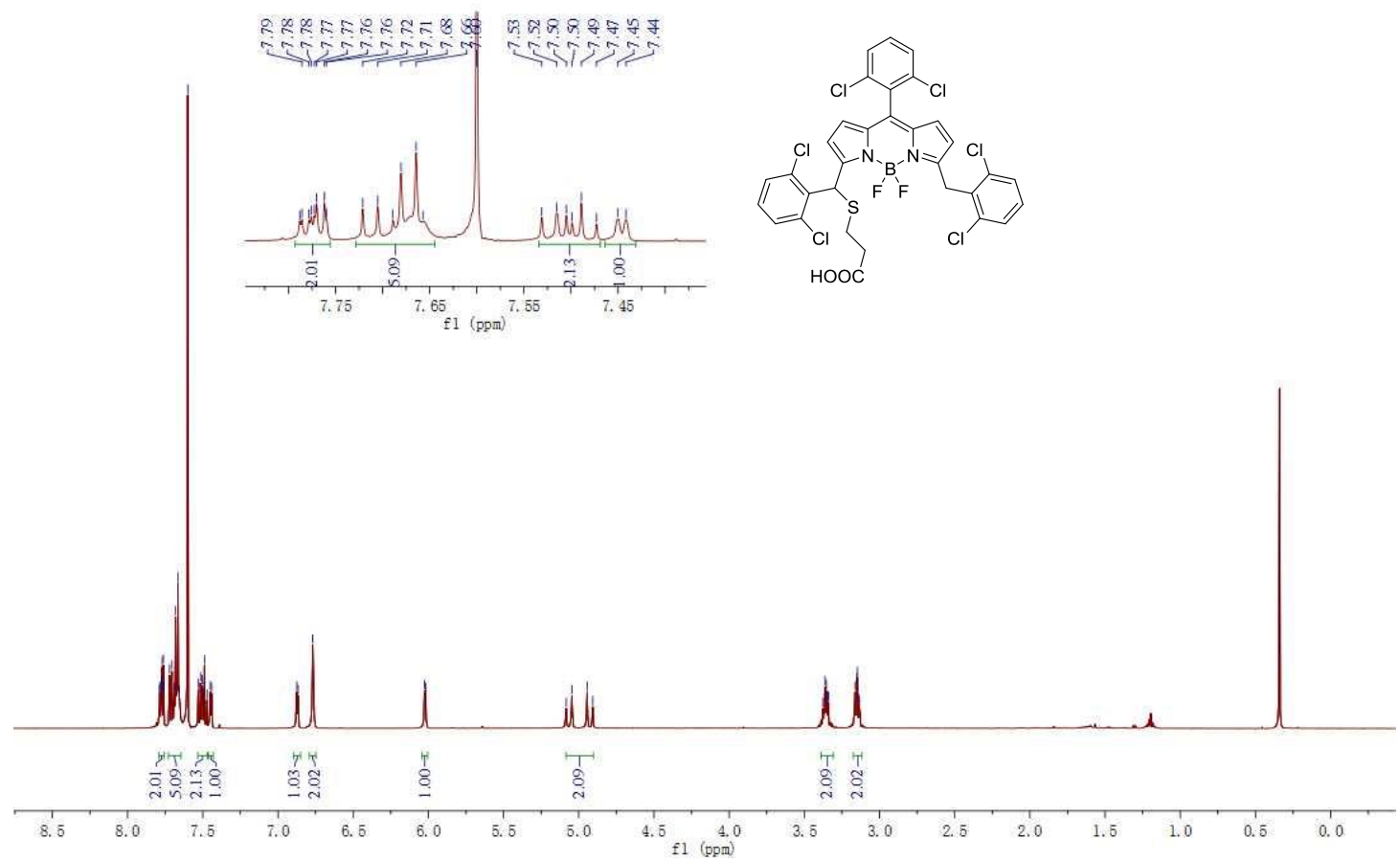

${ }^{1} \mathrm{H}$ NMR spectrum of $\mathbf{4 b}\left(\mathrm{CDCl}_{3}, 500 \mathrm{MHz}\right)$

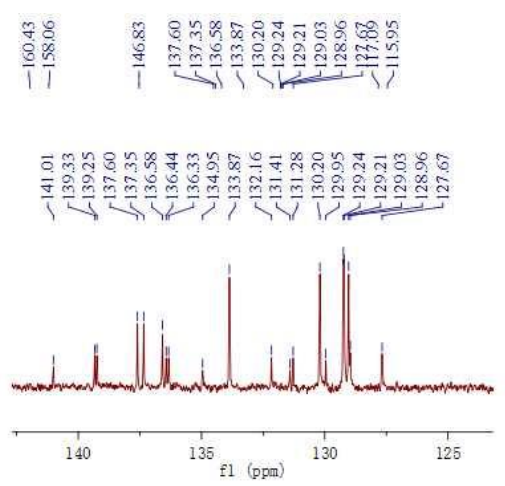

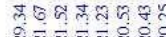

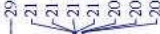
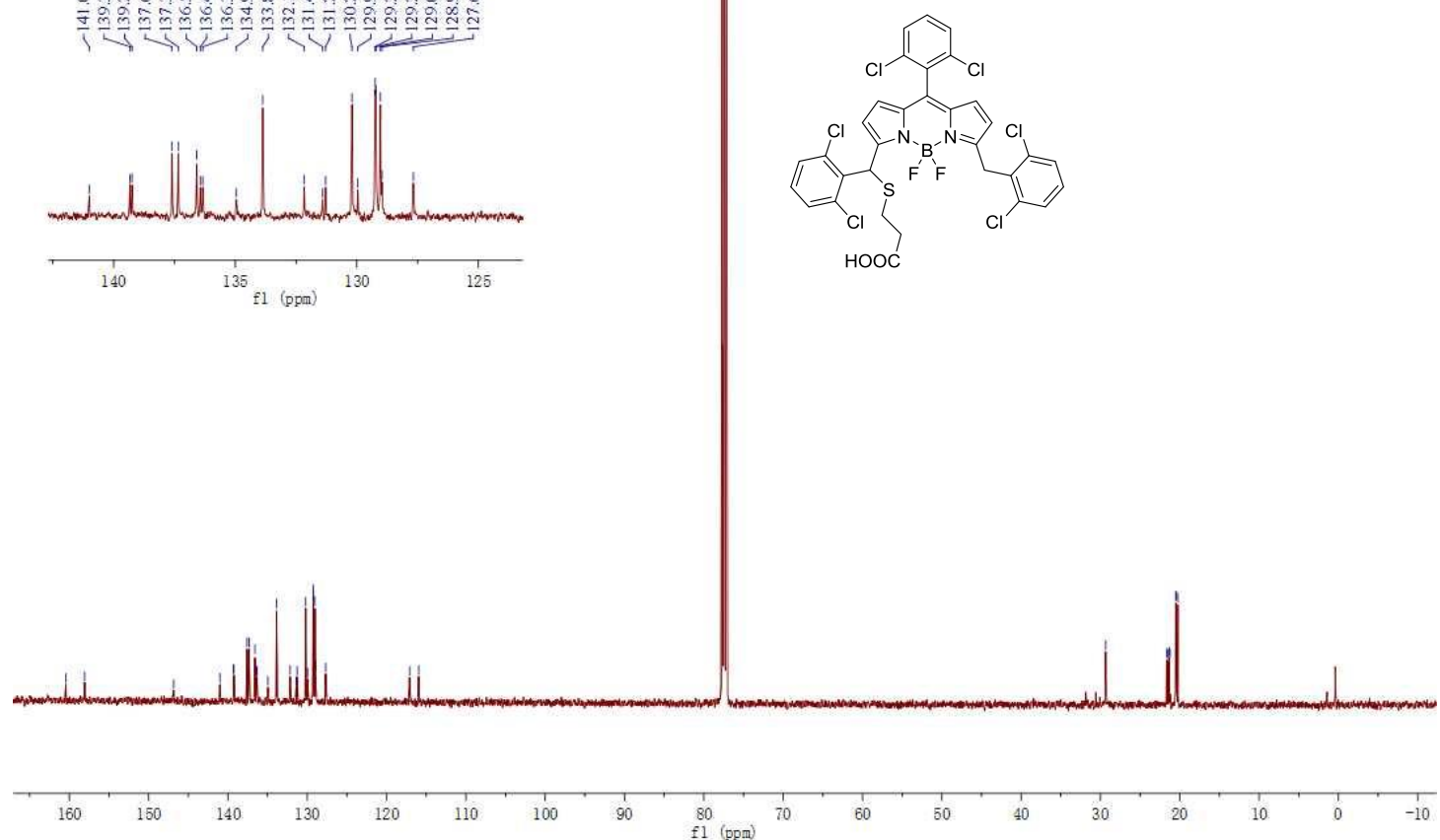

${ }^{13} \mathrm{C}\left\{{ }^{1} \mathrm{H}\right\}$ NMR spectrum of $\mathbf{4 b}\left(\mathrm{CDCl}_{3}, 126 \mathrm{MHz}\right)$ 


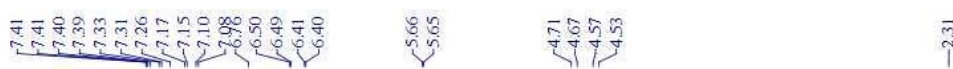
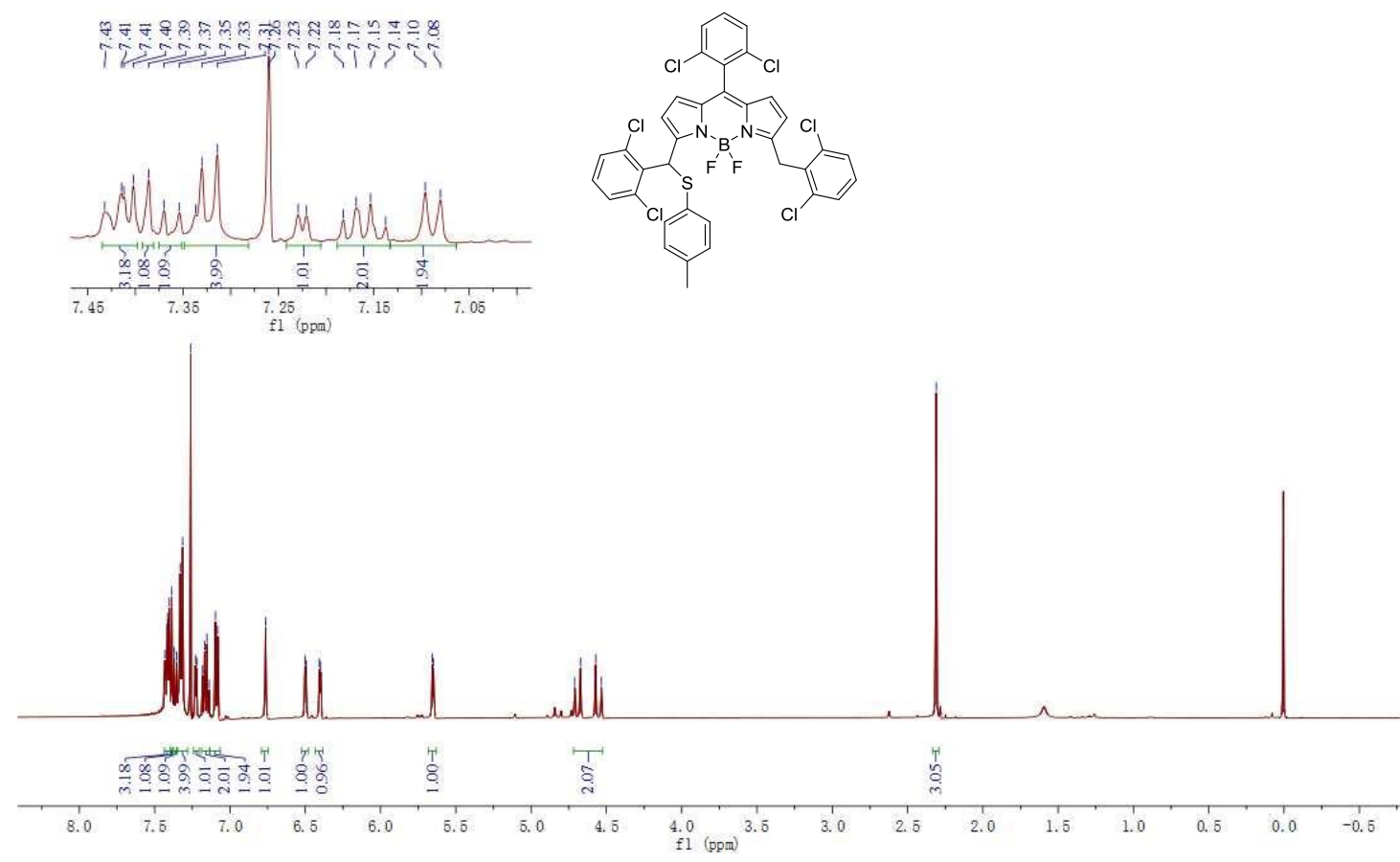

${ }^{1} \mathrm{H}$ NMR spectrum of $\mathbf{5 b}\left(\mathrm{CDCl}_{3}, 500 \mathrm{MHz}\right)$
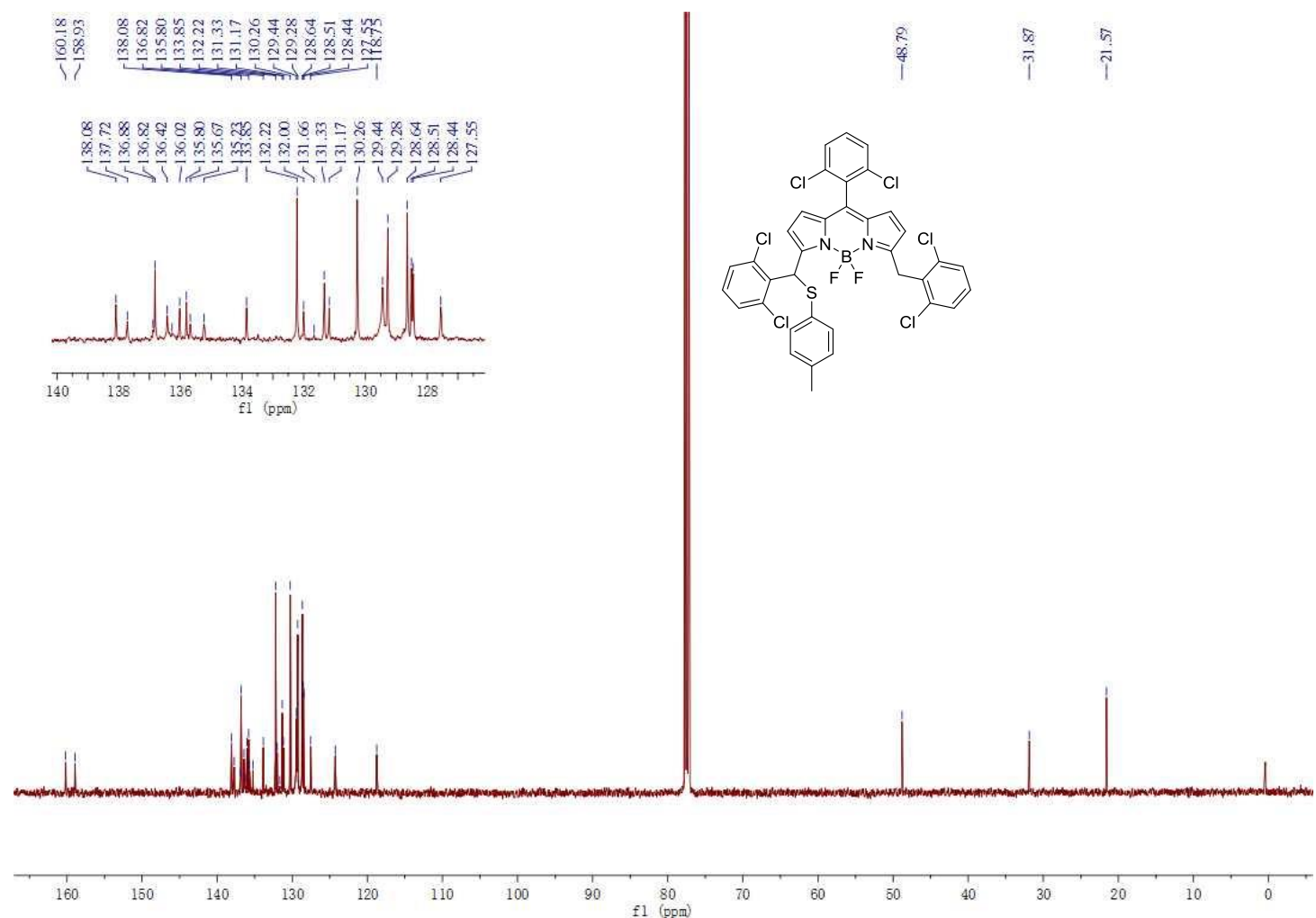

${ }^{13} \mathrm{C}\left\{{ }^{1} \mathrm{H}\right\}$ NMR spectrum of $\mathbf{5 b}\left(\mathrm{CDCl}_{3}, 126 \mathrm{MHz}\right)$ 


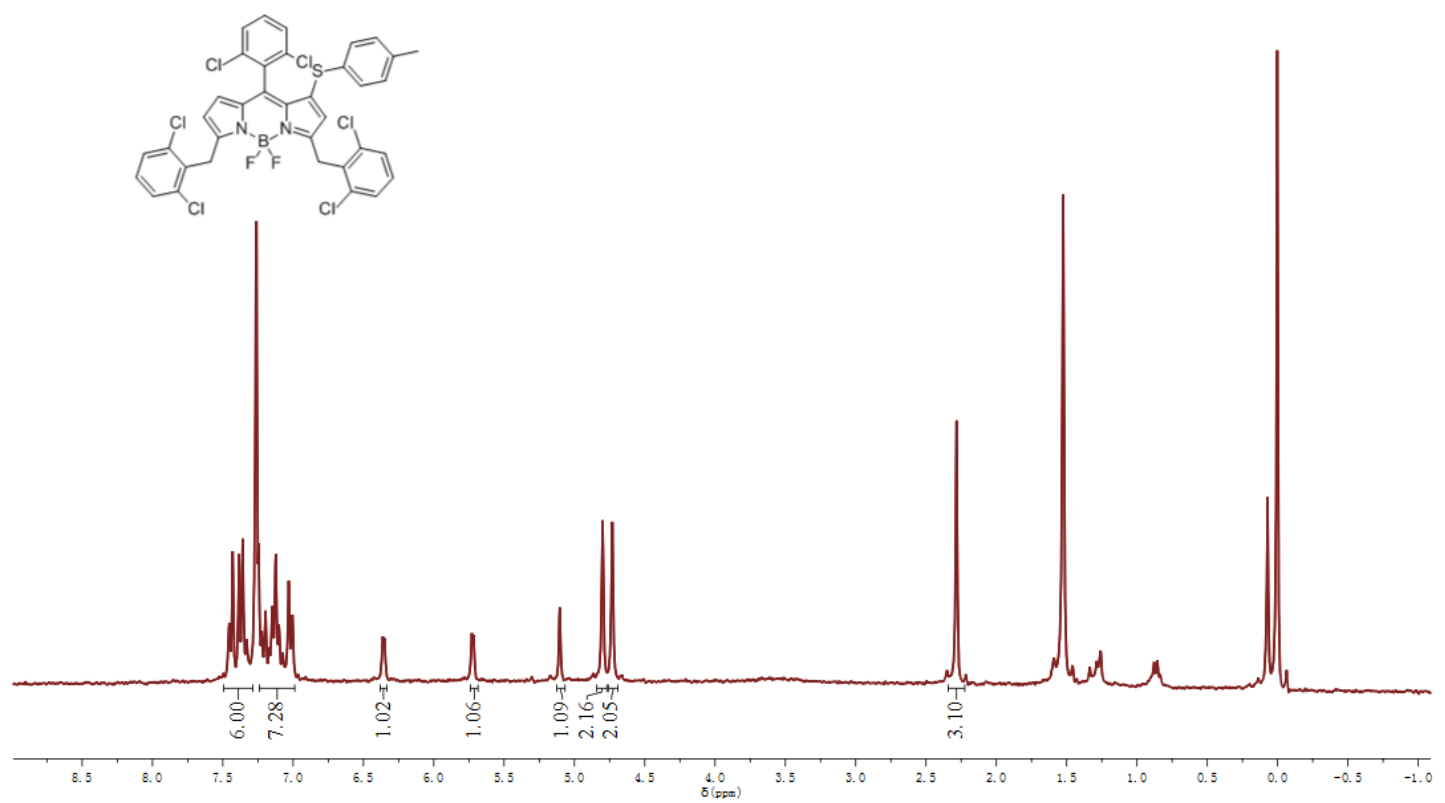

${ }^{1} \mathrm{H} \mathrm{NMR}$ spectrum of $\mathbf{6 b}\left(\mathrm{CDCl}_{3}, 300 \mathrm{MHz}\right)$
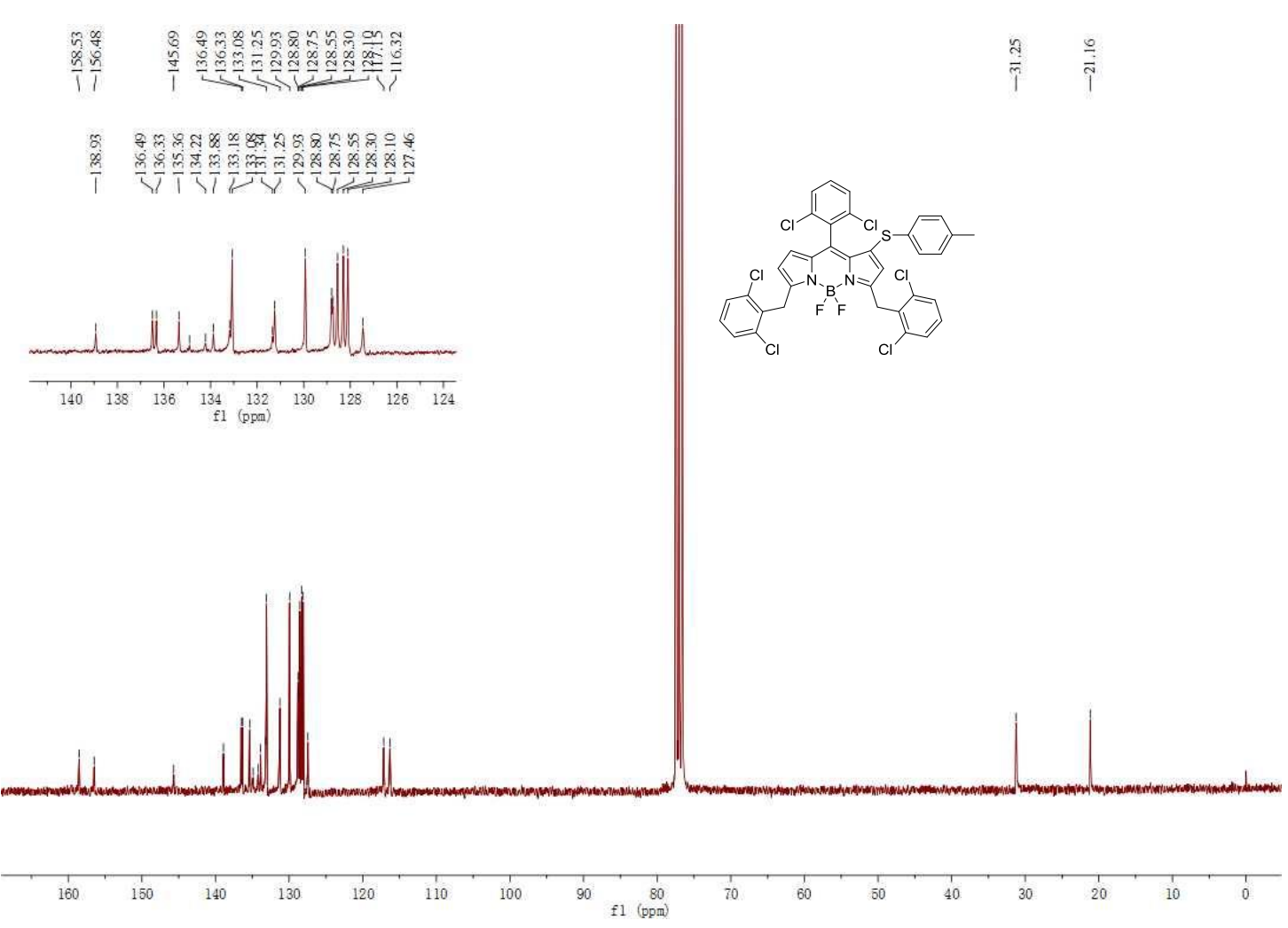

${ }^{13} \mathrm{C}\left\{{ }^{1} \mathrm{H}\right\}$ NMR spectrum of $\mathbf{6 b}\left(\mathrm{CDCl}_{3}, 75 \mathrm{MHz}\right)$ 


\section{High resolution mass spectroscopes for all new compounds}

2a HRMS (APCI) m/z: $[\mathrm{M}+\mathrm{H}]^{+}$Calcd. for $\mathrm{C}_{38} \mathrm{H}_{40} \mathrm{BF}_{2} \mathrm{~N}_{2}$ 573.3247; Found 573.3248.

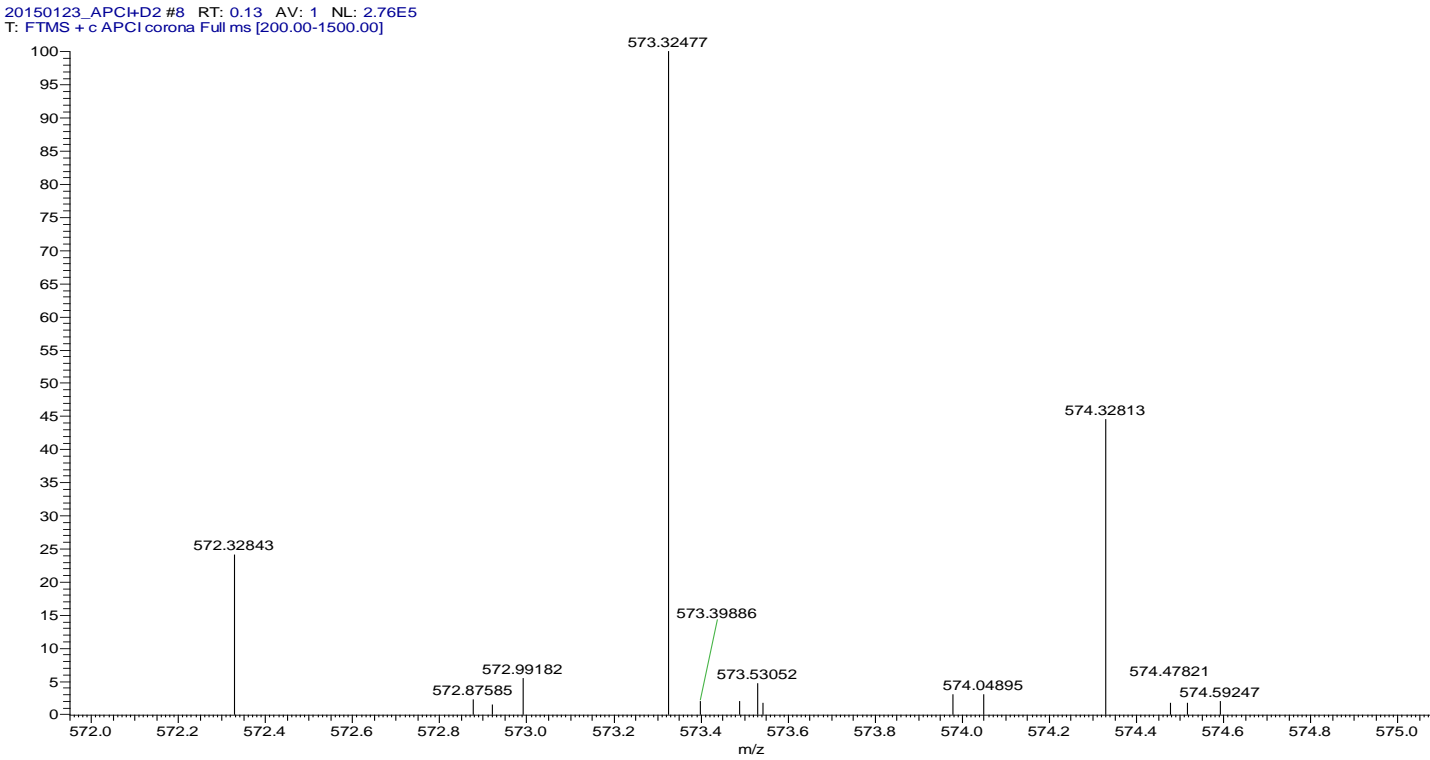

2b HRMS (APCI) m/z: [M - F] ${ }^{+}$Calcd. for $\mathrm{C}_{29} \mathrm{H}_{15} \mathrm{BCl}_{6} \mathrm{FN}_{2}$ 630.9438; Found 630.9433.

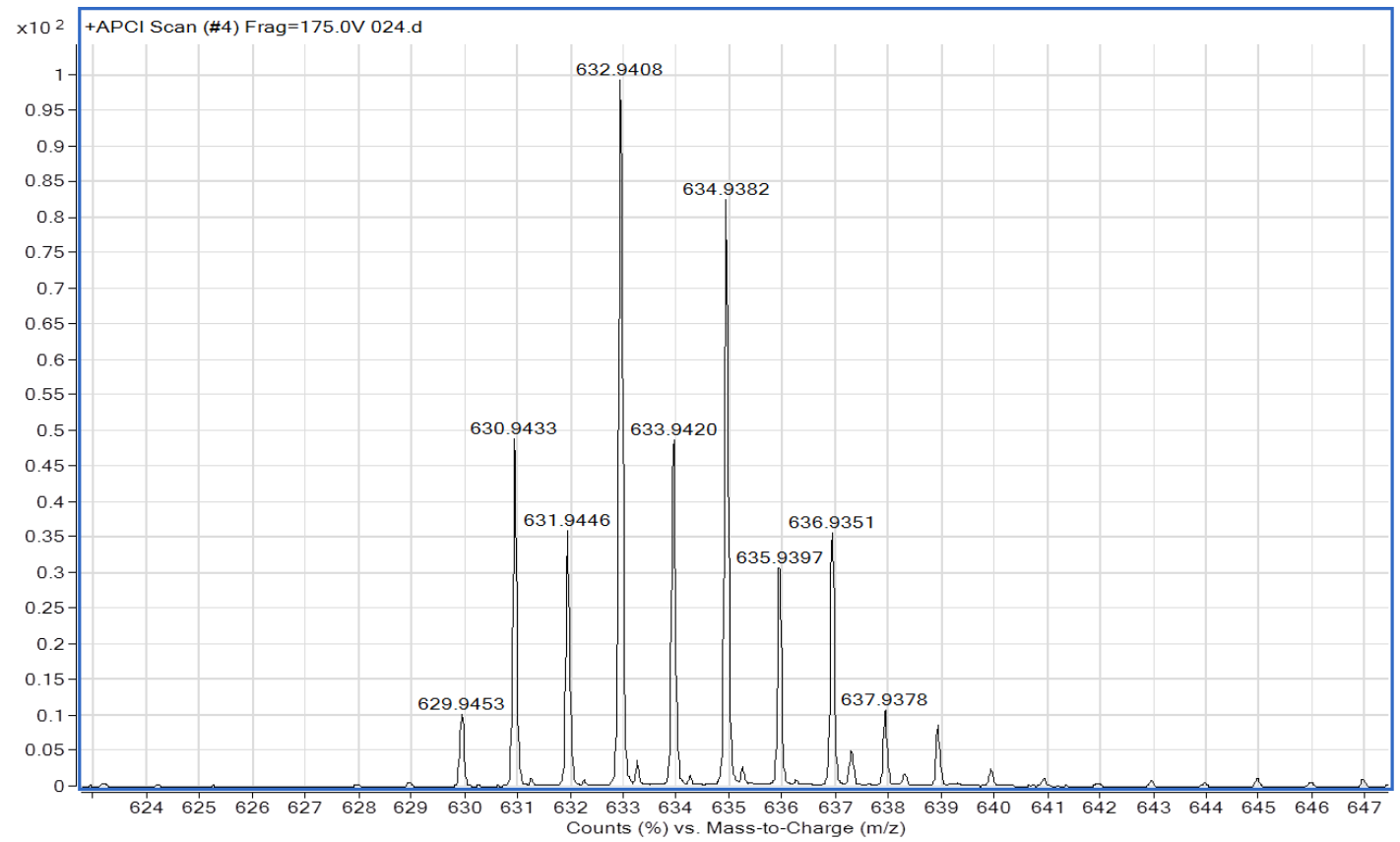


3a HRMS (ESI) m/z: $[\mathrm{M}+\mathrm{H}]^{+}$Calcd. for $\mathrm{C}_{46} \mathrm{H}_{51} \mathrm{BF}_{2} \mathrm{~N}_{3}$ 694.4139; Found 694.4132.

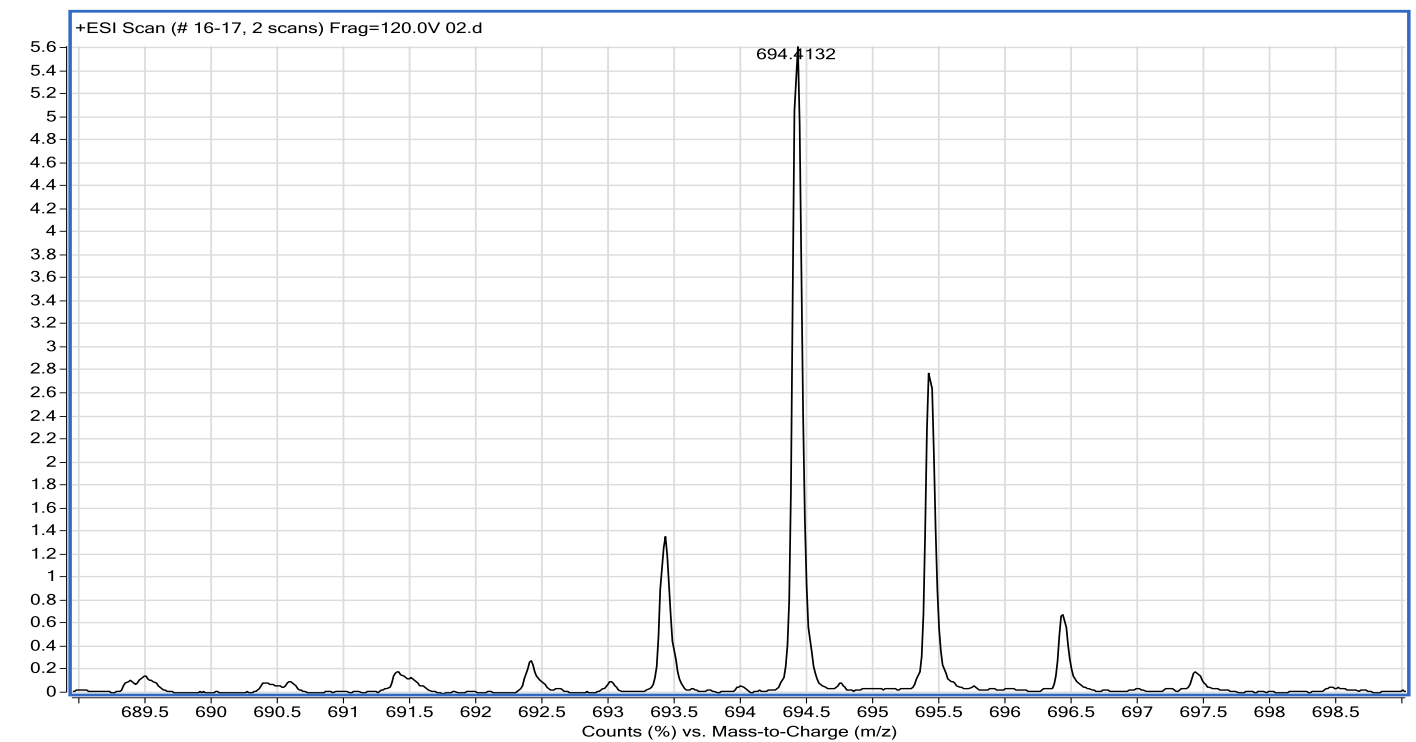

4a HRMS (APCI) m/z: [M - H] $]^{-}$Calcd. for $\mathrm{C}_{41} \mathrm{H}_{44} \mathrm{BF}_{2} \mathrm{~N}_{2} \mathrm{O}_{2} \mathrm{~S}$ 677.3179; Found 677.3165 .

20150123_APCI+D3 \#8 RT: 0.13 AV: 1 NL: 6.58 E
T: FTMS+c APCI corona Full ms [200.00-1500.00]

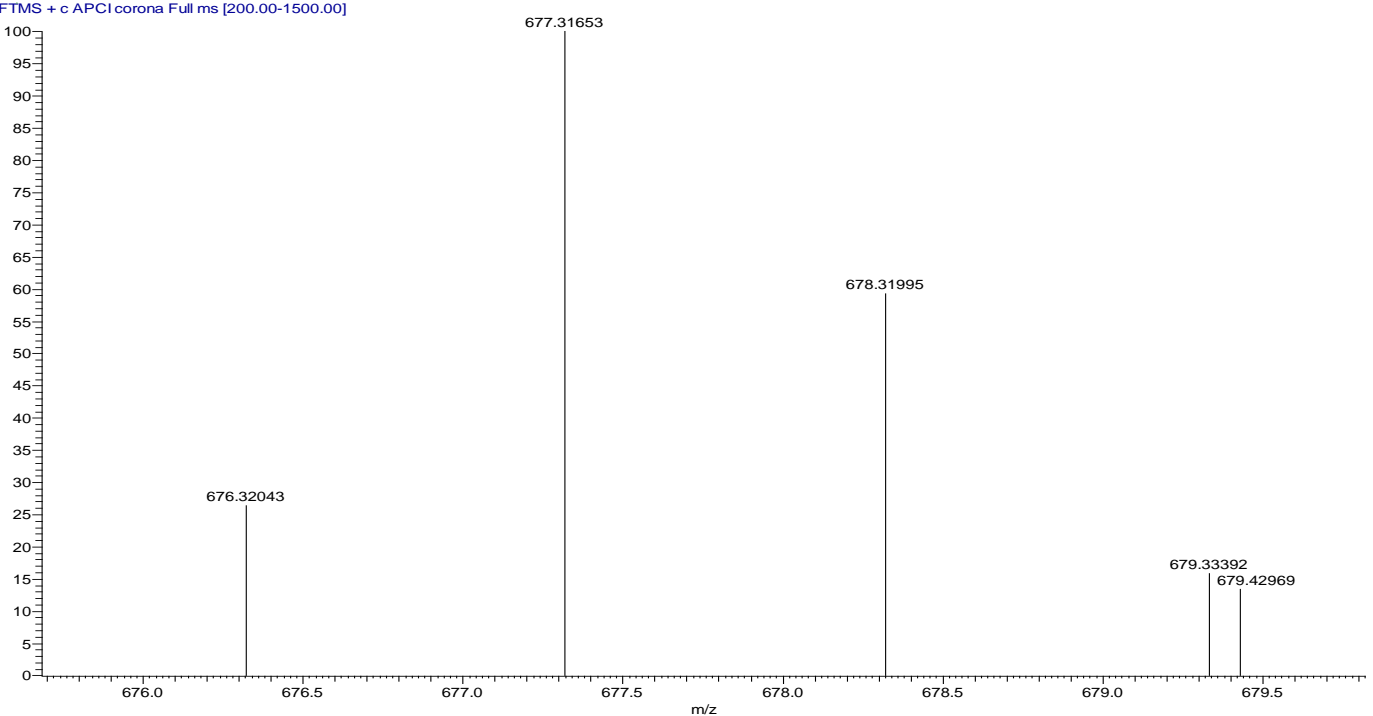


5a HRMS (APCI) m/z: [M - F $]^{+}$Calcd. for $\mathrm{C}_{45} \mathrm{H}_{47} \mathrm{BFN}_{2} \mathrm{~S}$ 677.3532; Found 677.3528.

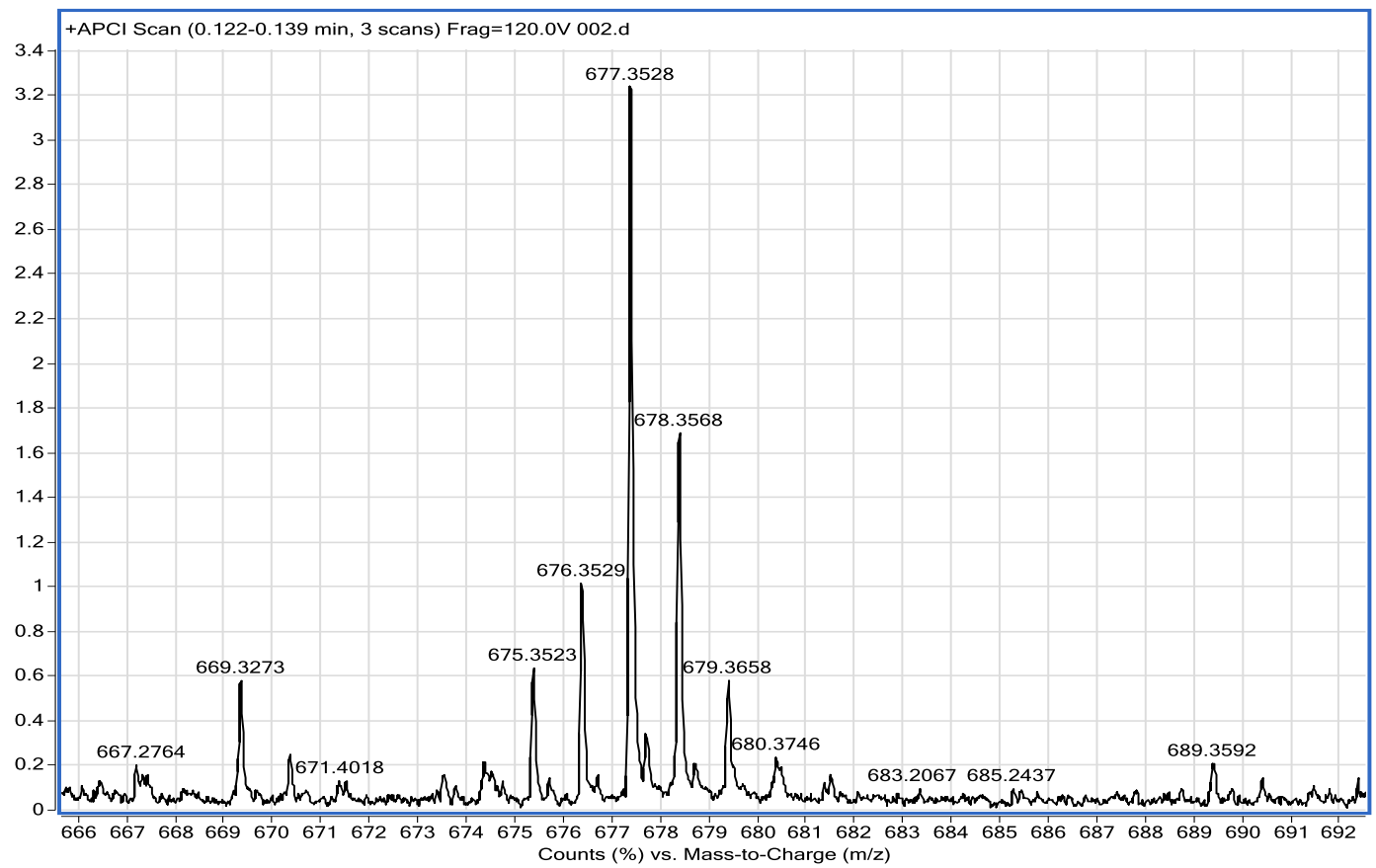

6a HRMS (APCI) m/z: [M - F] $]^{+}$Calcd. for $\mathrm{C}_{45} \mathrm{H}_{47} \mathrm{BFN}_{2} \mathrm{~S}$ 677.3537; Found 677.3530.

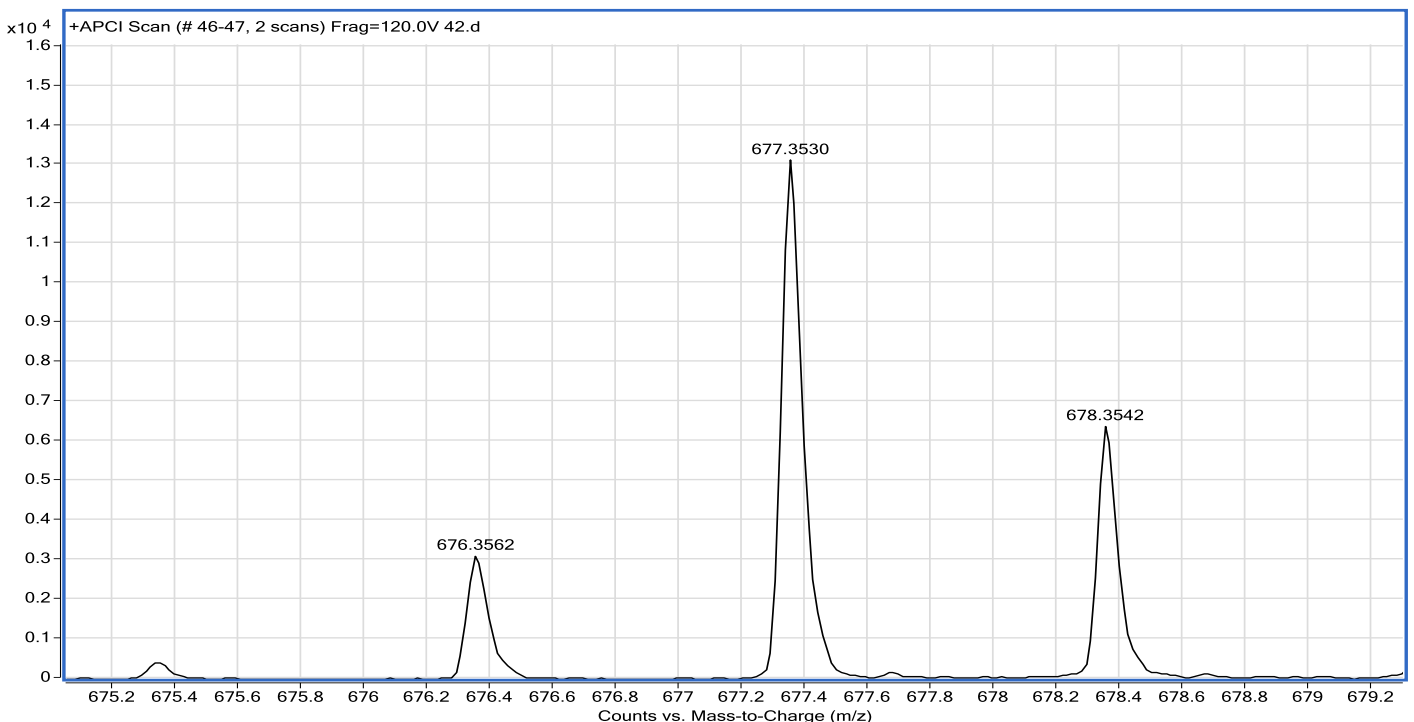


3b HRMS (ESI) m/z: $[\mathrm{M}+\mathrm{H}]^{+}$Calcd. for $\mathrm{C}_{37} \mathrm{H}_{27} \mathrm{~B}^{37} \mathrm{Cl}_{6} \mathrm{~F}_{2} \mathrm{~N}_{3}$ 774.0362; Found 774.0331 .

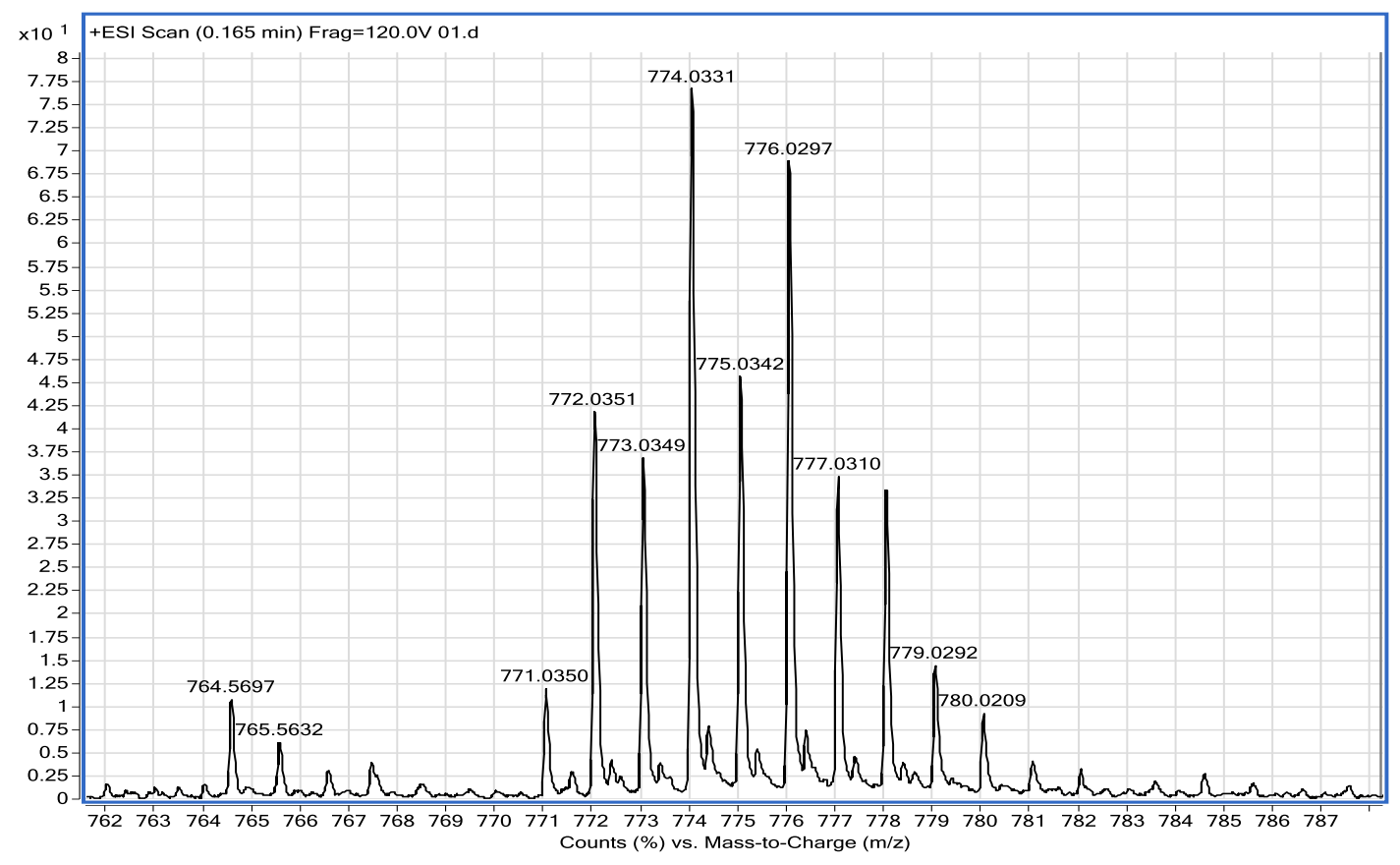

4b HRMS (MALDI) m/z: [M - H] $]^{-}$Calcd. for $\mathrm{C}_{32} \mathrm{H}_{20} \mathrm{~B}^{37} \mathrm{Cl}_{6} \mathrm{~F}_{2} \mathrm{~N}_{2} \mathrm{O}_{2} \mathrm{~S}$ 756.9422; Found 756.9415 .

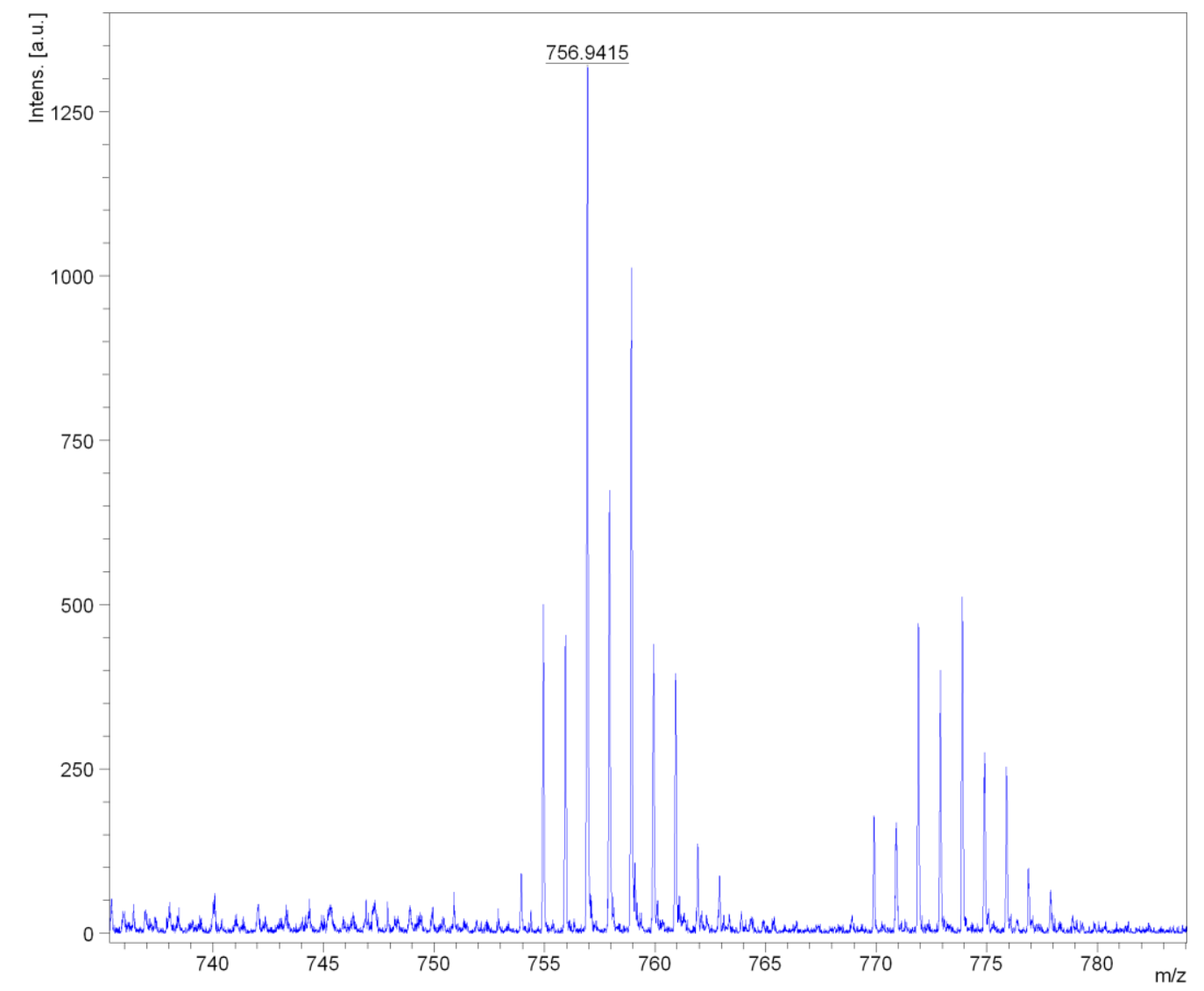


5b HRMS (APCI) m/z: [M - F] ${ }^{+}$Calcd. for $\mathrm{C}_{36} \mathrm{H}_{23} \mathrm{BCl}_{6} \mathrm{FN}_{2} \mathrm{~S}$ 754.9785; Found 754.9766 .

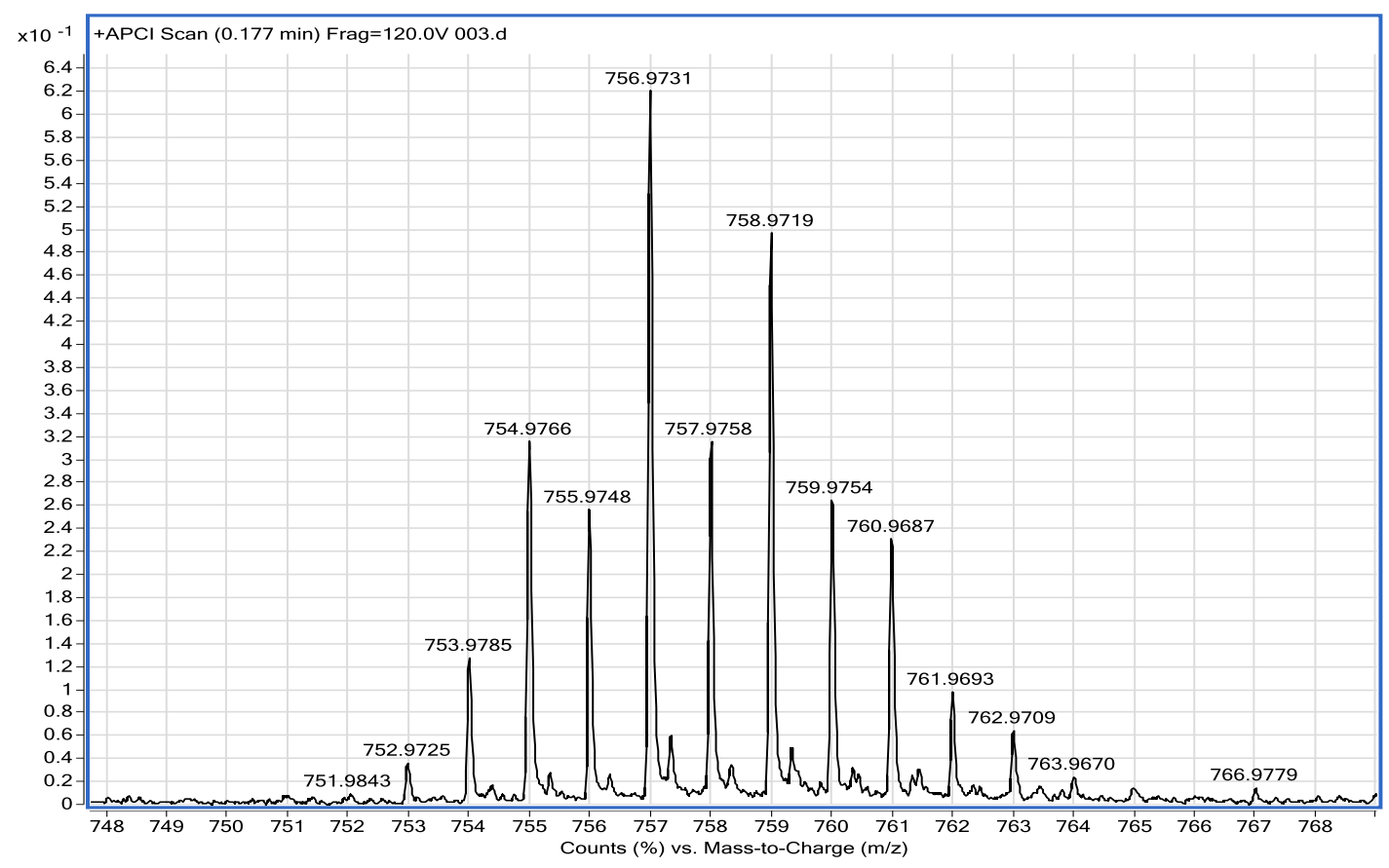

6b HRMS (APCI) m/z: [M - F] ${ }^{+}$Calcd. for $\mathrm{C}_{36} \mathrm{H}_{23} \mathrm{BCl}_{6} \mathrm{FN}_{2} \mathrm{~S}$ 754.9785; Found 754.9767.

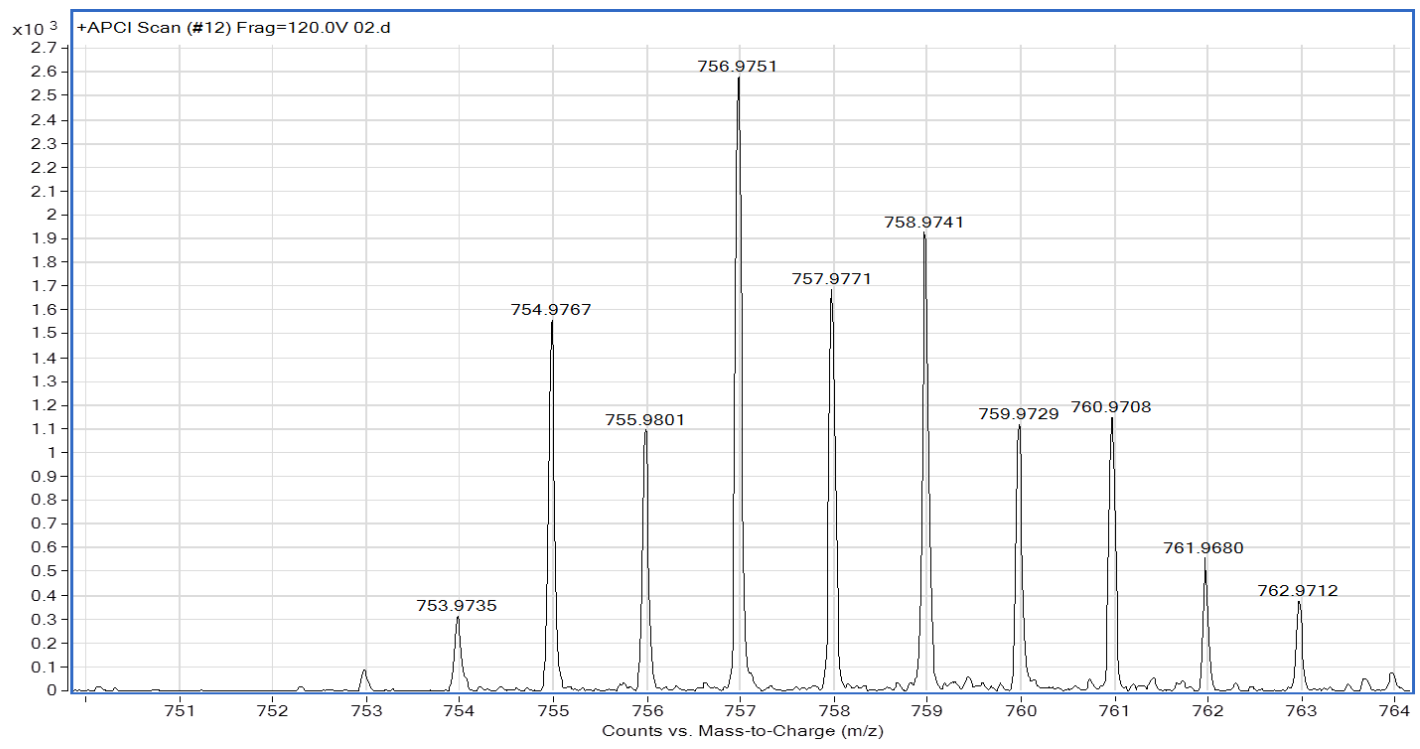


7 HRMS (APCI) m/z: $[\mathrm{M}+\mathrm{H}]^{+}$calcd. for $\mathrm{C}_{29} \mathrm{H}_{18} \mathrm{~B}^{37} \mathrm{Cl}_{6} \mathrm{~F}_{2} \mathrm{~N}_{2}$ 654.9633; Found 654.9639 .

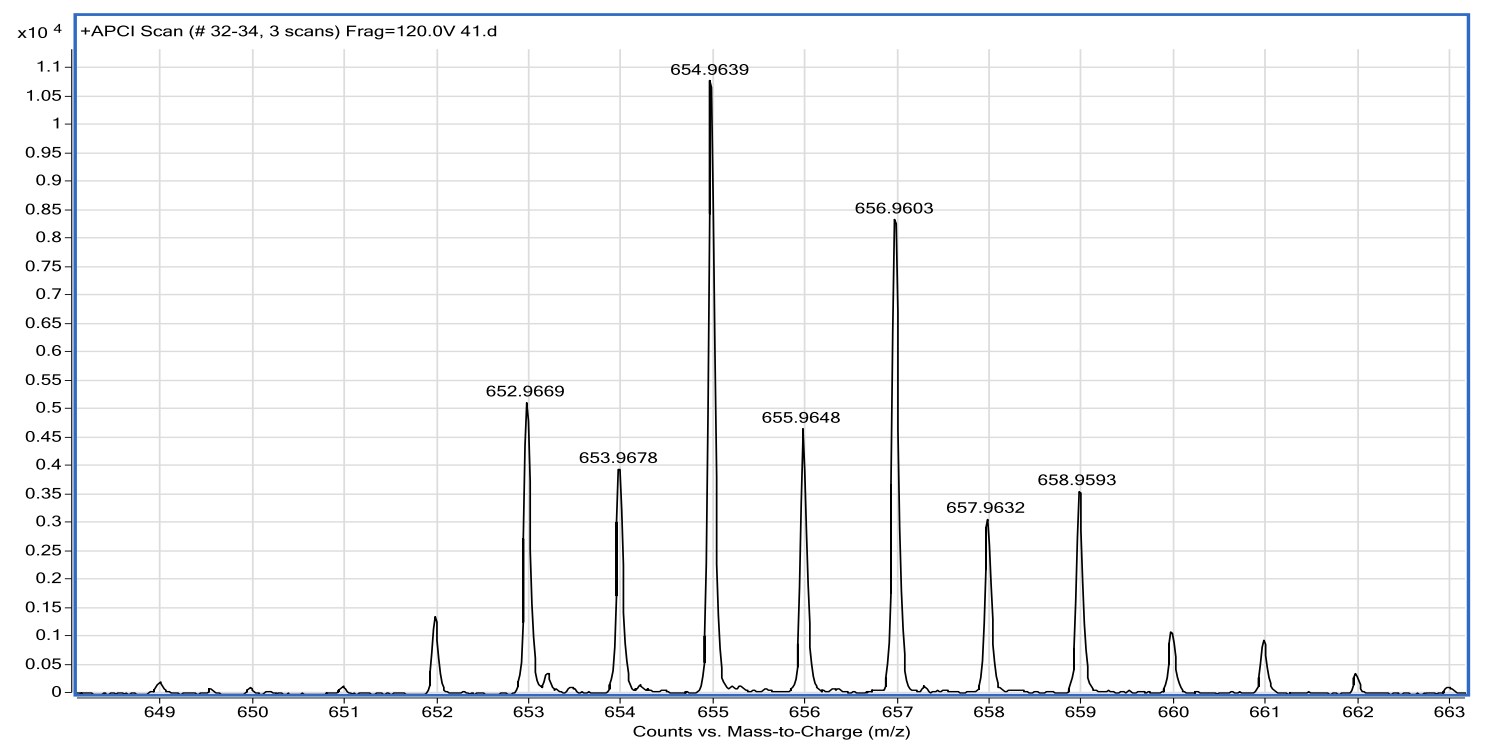

